\title{
负载型过渡金属催化剂在碳一碳键偶联反应中的应用研究进展
}

\author{
刘杰 ${ }^{a}$ 朱庆仁 ${ }^{a}$ 杜 娟 ${ }^{b}$ 张袖丽*,b \\ ( ${ }^{a}$ 淮北师范大学信息学院 淮北 235000)

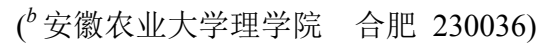

\begin{abstract}
摘要 过渡金属催化的偶联反应是有机合成中碳一碳键形成的重要方法, 也是当前有机化学研究领域的热点之一. 直 接以过渡金属盐或配合物作催化剂有价格昂贵、不易回收、痕量残留、污染产物和环境等弊端. 在环境问题倍受关注 的今天, 寻找绿色的物质转化途径, 开发可循环使用的负载型高效催化剂已成为化学家解决上述问题的重要途径之一. 按照载体分类, 综述了负载型催化剂在碳-碳键偶联反应中的研究进展.
\end{abstract}

关键词＼cjkstart过渡金属; 负载催化剂; 绿色化学; 催化剂分离回收

\section{Advances in Carbon-Carbon Coupling Reactions Catalyzed by Supported Transition-Metal Catalysts}

\author{
Liu, Jie $^{a} \quad$ Zhu, Qingren ${ }^{a} \quad$ Du, Juan ${ }^{b} \quad$ Zhang, Xiuli*,b \\ ( ${ }^{a}$ College of Information Science, Huaibei Normal University, Huaibei 235000) \\ ( ${ }^{b}$ College of Science, Anhui Agricultural University, Hefei 230036)
}

\begin{abstract}
The transition-metal catalyzed coupling reaction is one of the versatile methods for the generation of new carboncarbon bonds in organic synthesis, and it has become a hot topic in current organic chemistry. Transition metal salts and their complexes were used as catalyst directly in the classic methods with the drawbacks of high cost of the transition metal, the metal contamination of the product and the toxicity remained. In order to overcome these problems, developing green synthetic methods based on efficient and reusable catalytic systems is more desirable in organic synthesis. In this paper, the recent progress on the carbon-carbon coupling reactions catalyzed by the supported transition-metal catalysts has been reviewed.
\end{abstract}

Keywords transition metals; supported catalysts; green chemistry; separation and recovery of catalyst

在过去几十年里, 过渡金属催化的均相有机合成反 应受到了化学家的广泛关注并引起人们的极大兴趣, 它 改变了传统的有机合成方法, 大大缩短了合成路径, 因 而过渡金属催化的均相有机合成反应一直占据着有机 合成领域的主导地位 ${ }^{[1]}$. 但是均相催化体系存在一些制 约其发展的瓶颈问题, 如: 过渡金属催化剂的价格较为 昂贵、不能重复使用、产物分离困难以及产物中含有痕 量过渡金属催化剂残留等问题. 随着药物合成化学和有 机化学等对洁净化合成的严格要求, 可用于液相合成的 固载化催化剂愈来愈受到人们的青睐, 这使得合成化学 家们把更多的兴趣转移到可以回收再利用并且容易分 离的负载型催化剂上, 开发负载型过渡金属催化剂是解 决均相催化剂较难分离与回收以及过渡金属残留等问
题的主要策略之一. 载体需选用具有来源广泛, 价廉易 得, 溶剂适用性广, 具有较好的热稳定性和机械强度, 易于纯化和回收等优点的无机、有机或杂化材料. 另外 催化剂载体的使用为反应提供了一种全新的反应环境, 为进一步研究催化反应历程提供了很好的思路. 近年来 许多负载型金属催化剂由此被设计合成, 并对很多反应 体系起到了较好的催化效果 ${ }^{[2]}$, 有些催化剂已经表现出 了具有工业应用的潜在价值. 成功的负载型催化剂在反 应中可以催化量使反应进行完全, 其效果与均相催化体 系效果相当，而且反应结束后，通过简单分离除去溶剂 后即可得到产物, 并回收可循环使用的载体催化剂(图 1).

负载型过渡金属催化剂的制备方法主要分为两种,

\footnotetext{
*E-mail: zhxiuli@163.com

Received August 13, 2014; revised October 7, 2014; published online October 16, 2014.

Project supported by the Natural Key Project of Education Department of Anhui Province (No. KJ2013A122).

安徽省教育厅自然科学重点(No. KJ2013A122)资助项目.
} 


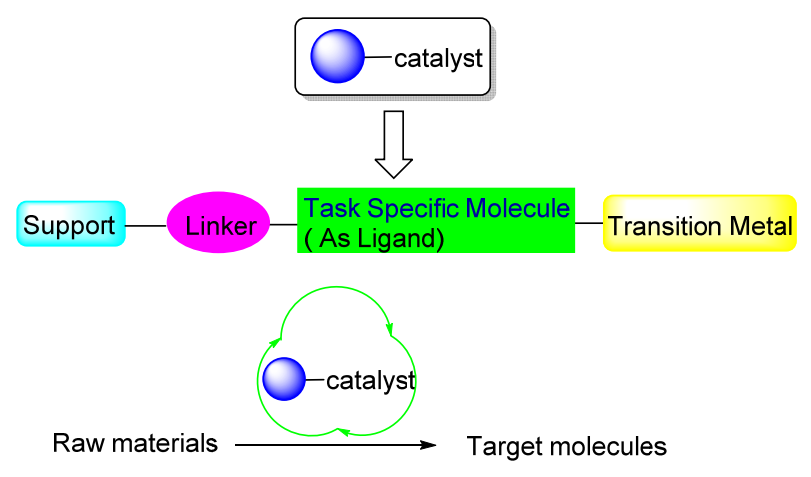

图 1 负载催化剂的设计策略和催化作用

Figure 1 Design and application of the supported catalyst

一种是通过将过渡金属或者金属配合物吸附于载体表 面上来实现, 另外一种是通过化学键联的手段, 先在载 体表面上接枝具有特殊功能的基团, 再经由配位方式将 过渡金属载于功能基团上. 根据载体种类的不同, 本文 对近年来负载型催化剂及其催化的碳一碳键偶联反应研 究进展进行分类介绍.

\section{1 无机氧化物载体负载过渡金属催化剂}

一些无机载体, 如金属氧化物, 可以负载过渡金属 作为非均相催化剂来催化碳一碳键的偶联反应, 取得了 好的效果. Köhler 小组 ${ }^{[3]}$ 曾报道在无机氧化物(如 $\mathrm{MgO}$ 、 $\mathrm{TiO}_{2} 、 \mathrm{Al}_{2} \mathrm{O}_{3}$ 等)表面负载的钯试剂催化的对位取代的澳 苯与苯乙烯的偶联反应(Eq. 1), 其中芳烃取代基的电子 效应及烯烃 $\pi$ 键的电子密度对反应的进行有很大的影 响. 但是由于载体表面和钯之间没有牢固的化学键相 连, 致使在循环使用过程中钯的流失量较大, 因而催化 剂的催化活性随循环次数增加降低很快. Davies 等 ${ }^{\left[{ }^{[}\right]}$较 深入地研究了三氧化二铝负载钯催化剂 $\left(\mathrm{Pd} / \mathrm{Al}_{2} \mathrm{O}_{3}\right)$ 进行
的 Heck 反应体系，首次提出了非均相钯基催化体系的 均相催化机理(非均相钯的均相化反应).

$$
\begin{aligned}
& \mathrm{PhBr}+\mathrm{PhHC}=\mathrm{CH}_{2} \frac{\mathrm{Pd} / \mathrm{MO}_{x}(0.1 \mathrm{~mol} \%)}{\mathrm{NaOAc}, \mathrm{DMA}, 140^{\circ} \mathrm{C}} \\
& \mathrm{MO}_{x}=\mathrm{MgO}, \mathrm{TiO}_{2}, \mathrm{Al}_{2} \mathrm{O}_{3}, \mathrm{SiO}_{2}
\end{aligned}
$$

Iyer 等 ${ }^{[5]}$ 尝试采用价格相对价廉的金属镍，制备了 $\mathrm{Ni}(0) / \mathrm{Al}_{2} \mathrm{O}_{3}$ 负载型催化剂, 在碘代芳烃和丙烯酸乙酯或 苯乙烯的 Heck 反应中表现出一定的催化活性, 但不能 催化溴代或氯代芳烃的 Heck 反应.

1999 年, Kabalka 课题组 ${ }^{[6]}$ 将 $\mathrm{Pd}(0)$ 掺入 $\mathrm{KF} / \mathrm{Al}_{2} \mathrm{O}_{3}$ 催 化体系应用于无配体加入、无溶剂的 Suzuki 偶联反应中. 实验表明在此体系中，碘代芳烃以及澳代芳烃均能有较 好的收率, 氯代芳烃的反应效果不佳, 芳基硼酸要比烯 烃嗍酸效果更好, 但该体系的不足之处是钯催化剂的用 量较大.

2002 年, Barbarella 等 ${ }^{[7]}$ 使用 $\mathrm{PdCl}_{2}(\mathrm{dppf})_{2}$ 作为钯源, 将其负载到 $\mathrm{KF} / \mathrm{Al}_{2} \mathrm{O}_{3}$, 在无溶剂条件下实现了微波促进 的 Suzuki 偶联反应(Scheme 1). 该方法大大加快了反应 速率, 仅需要几分钟即可完成反应. 同时为研究高纯度 的噻吩低聚物的合成提供了一种快速、高效而且对环境 友好的方法，可制备高产率的预期产物.

2012 年, $\mathrm{Li}$ 和 Chang 等 ${ }^{[8]}$ 首次报道了氧化铝固载纳 米钯催化的芳基硼酸和芳基重氮盐的 Suzuki 偶联反应 (Scheme 2), 反应不需要额外添加碱和配体, 以甲醇为 溶剂, 室温下即可进行.

\section{2 活性炭负载过渡金属催化剂}

活性炭具有发达的孔隙结构、巨大的比表面和优越 的吸附性能, 化学性质稳定, 不溶于有机溶剂, 是一类

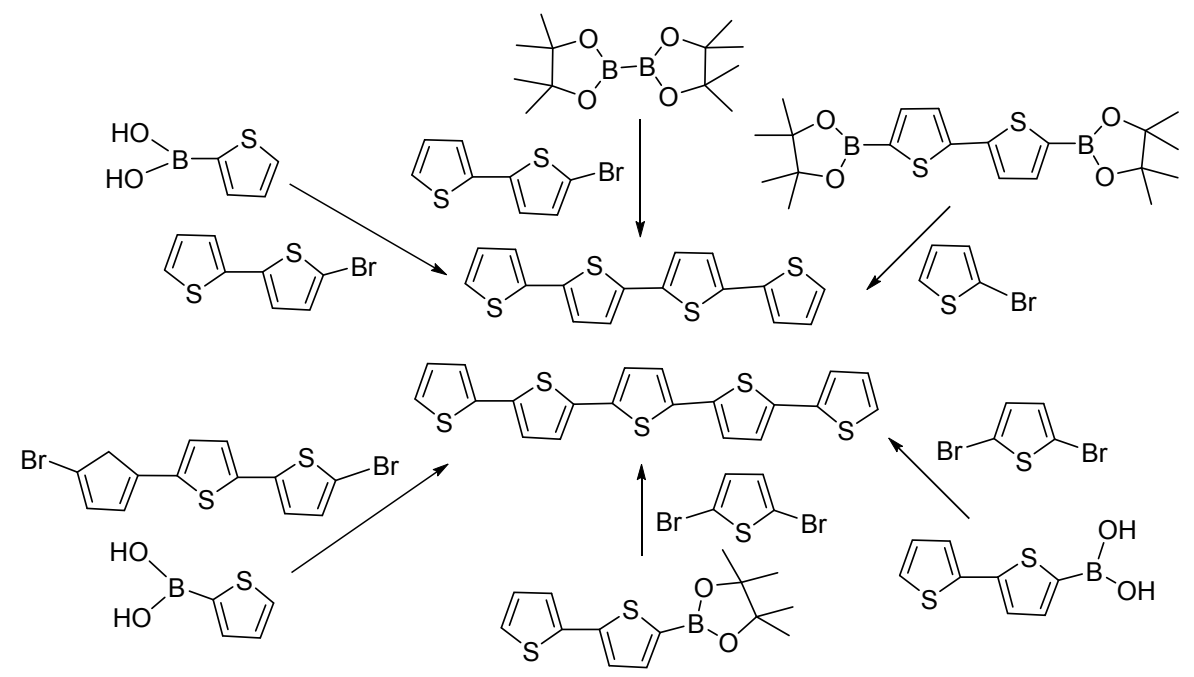

图式 $1 \mathrm{KF} / \mathrm{Al}_{2} \mathrm{O}_{3}$ 负载钯在无溶剂下微波促进的 Suzuki 偶联反应

Scheme 1 Pd doped on $\mathrm{KF} / \mathrm{Al}_{2} \mathrm{O}_{3}$ catalyzed Suzuki reaction under microwave irradiation and solvent-free conditions 


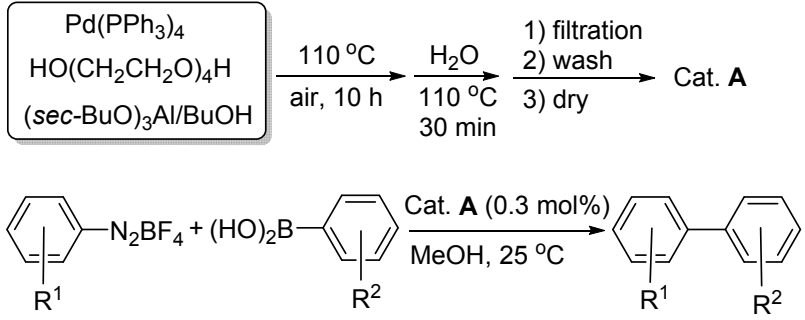

图式 2 芳基嗍酸和芳基重氮盐的 Suzuki 偶联反应

Scheme 2 Suzuki reaction of arylboronic acids and arenediazonium salts

非常理想的催化剂载体, 目前已广泛用于负载多种过渡 金属, 如 $\mathrm{Au} / \mathrm{C}, \mathrm{Pd} / \mathrm{C}, \mathrm{Ni} / \mathrm{C}, \mathrm{Cu} / \mathrm{C}$ 等催化剂已成功应用于 有机合成反应，其中 $\mathrm{Pd} / \mathrm{C}$ 催化剂的应用最为广泛.

1994 年, Marck 等 ${ }^{[9]}$ 报道了 $\mathrm{Pd} / \mathrm{C}$ 催化的 Suzuki 偶联 反应. 当以溴代芳烃和三氟磺酸酯为反应底物时, 可得 到较好的结果 (Scheme 3). 同时, 作者研究了配体对 $\mathrm{Pd} / \mathrm{C}$ 催化体系活性的影响. 结果表明, 当以溴代芳烃为 底物时，同样可获得较高反应收率，但是当使用芳基氯 代物时, 产率较低.
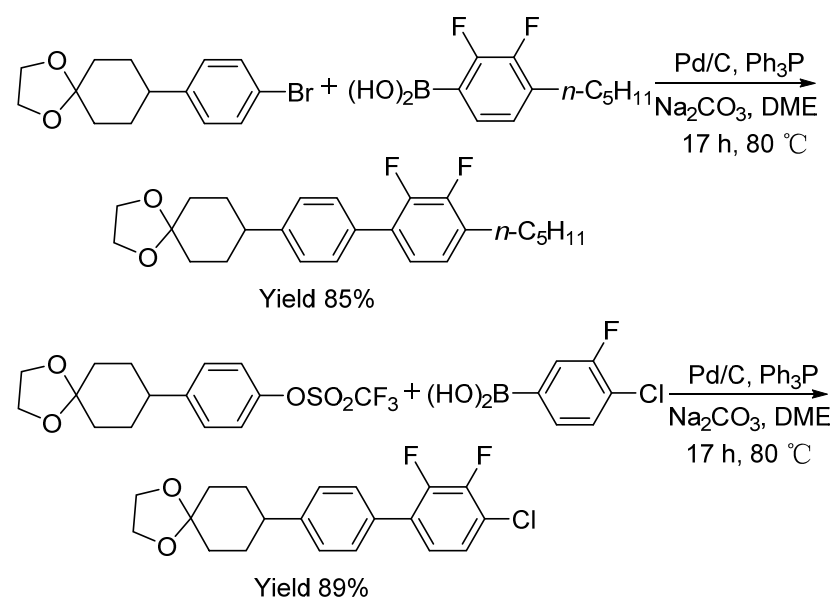

图式 3 溴代芳烃和三氟磺酸酯与芳基硼酸的 Suzuki 反应

Scheme 3 Suzuki reaction of arylbromides and aryltriflates with organoboronic acids

Köhler 小组 ${ }^{[10]}$ 制备了系列不同的 $\mathrm{Pd} / \mathrm{C}$ 催化剂, 并 以 Heck 反应为模板, 全面研究了催化剂中 $\operatorname{Pd}(0)$ 颗粒分 散度、还原程度、含水量、负载量等因素对催化剂活性 的影响, 实验发现纳米钯颗粒分散比较均匀, 低还原度, 含水量在 $50 \% \sim 60 \%$ 的催化剂的催化活性最高. 此外, $\mathrm{Pd} / \mathrm{C}$ 催化剂在离子液体中 ${ }^{[11]}(\mathrm{Eq} .2)$ 和在超声作用下 ${ }^{[12]}$ 的低温 Heck 反应被相继被报道, 发现其均具有较高的 催化活性和重复使用性能.

2012 年, Sun 等 ${ }^{[13]}$ 报道了在 $50 \%$ 甲醇水溶液中 $\mathrm{Pd} / \mathrm{C}$ 催化的 18-冠-6 促进的芳基溴化物和芳基硼酸的 Suzuki 偶联反应(Eq. 3). 底物适用范围较广, 各种含有吸电或

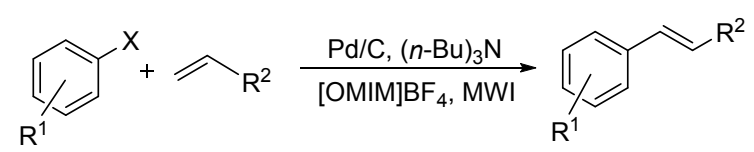

[OMIM] $\mathrm{BF}_{4}=1$-octanyl-3-methylimidazolium tetrafluoroborate $\mathrm{R}^{1}=\mathrm{H}, \mathrm{OCH}_{3}, \mathrm{NO}_{2} ; \mathrm{R}^{2}=\mathrm{Ph}, \mathrm{CO}_{2} \mathrm{C}_{2} \mathrm{H}_{5} ; \mathrm{X}=\mathrm{Br}, \mathrm{Cl}$

者供电基团的芳基溴化物和芳基喼酸都能高产率地顺 利进行，产率最高达 99\%。此外，该催化剂可以循环使 用 5 次而不丧失催化活性.

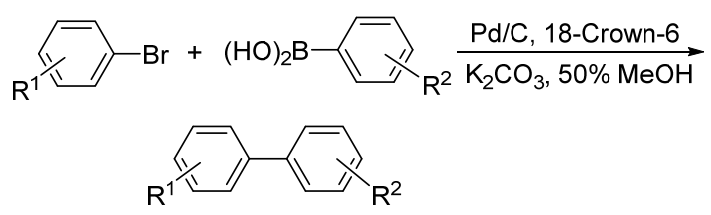

2013 年, Liu 等 ${ }^{[14]}$ 报道了无配体参与的 $\mathrm{Pd} / \mathrm{C}$ 催化的 芳基溴化物和芳基嗍酸的 Suzuki 偶联反应(Eq. 4). 在室 温条件下，各种取代基的芳基溴化物和芳基硼酸都能高 产率地在 $50 \%$ 乙醇水溶液中顺利完成, 催化剂可循环利 用 10 次后，其催化活性未见显著降低. 该方法可用于含 氟液晶化合物的合成.

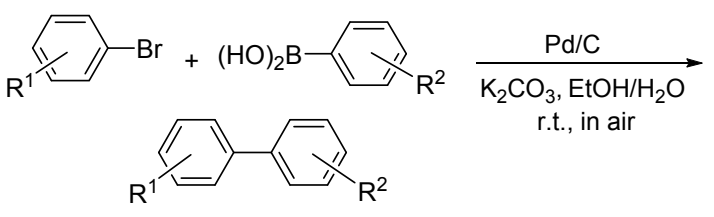

\section{3 有机载体负载过渡金属催化剂}

催化剂的载体除了简单无机载体以外，有机载体的 发展也比较迅速. 在有机载体中, 最常见的两种载体是 有机高分子聚合物和离子液体.

\section{1 有机高分子聚合物负载过渡金属催化剂}

有机高分子聚合物负载过渡金属催化剂一般是在 聚合物的单体中有目的地镶嵌，能够充当配体的原子或 官能团，或者在已成型的功能化聚合物上接枝修饰引入 配体, 再经由配位方式将过渡金属载于引入的配体上.

聚苯乙烯是常用的聚合物载体之一，早在 1978 年, Teranishi 等 ${ }^{[15]}$ 曾报道了聚苯乙烯经过负载过渡金属钯 而制得钯的配合物，并成功应用于催化 Heck 反应. 后 来, Zhang 小组 ${ }^{[16]}$ 也成功合成了接枝有邻菲洛林的聚苯 乙烯的载体，同样可以负载钯，并用于催化碘代芳烃和 丙烯酰胺的反应.

2000 年, Schwarz 课题组 ${ }^{[17]}$ 报道了在改性聚苯乙烯 表面的氧中心固定 $\mathrm{Pd}$ 配合物制得载体催化剂. 该催化 剂对丙烯酸酯与卤代芳香烃的 Heck 反应具有较高的催 化活性, 可高产率地催化丙烯酸丁酯与碘(溴)代芳烃的 反应; 也可以中等收率催化丙烯酸丁酯与氯代芳烃的反 
应; 此外, 催化剂在重复使用 15 次后, 其活性未见明显 降低.

尔后, Lin 等 ${ }^{[18]}$ 和 Yamada 等 ${ }^{[19]}$ 分别深入研究了聚合 物负载 Pd 催化剂的结构及其在 Heck 反应中的催化活 性.

Wang 小组 ${ }^{[20]}$ 以聚苯乙烯作为载体, 经过一步简单 反应将卡宾单元嫁接在载体表面, 得到负载型卡宾银催 化剂. 在室温和无溶剂的条件下, 将该催化剂用于醛、 炔和胺的三分子串联反应, 制备了系列炔丙胺类化合物 (Eq. 5). 催化剂可以循环使用 12 次, 其稳定性和活性均 无明显变化, 该工作被 Synfacts 作为亮点进行了评 论 ${ }^{[20 b]}$. 同年他们还报道了廉价金属铁及铟催化的该类 反应，并提出可能反应机理 ${ }^{[21]}$.

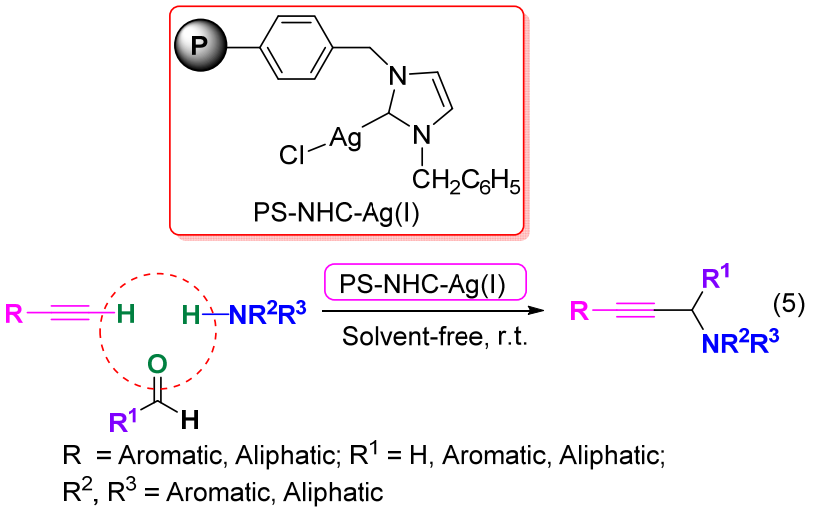

Billaud 课题组 ${ }^{[22]}$ 尝试采用价格相对便宜但同样具 有较高催化活性的过渡金属镍作为 Heck 反应催化剂的 活性组分. 将 Ni 颗粒分散于聚苯胺(PANI)中制备了 $\mathrm{Ni}(0) / \mathrm{PANI}$ 催化剂, 碘苯转化率可达为 $69 \%$, 催化剂稳 定性很好, 在反应结束后未发现金属镍的沉积.

Yang 等 ${ }^{[23]}$ 发现聚- $\gamma$-氨丙基硅烷上负载均相 $\mathrm{Co}(\mathrm{OAc})_{2}$ 亦可作为 Heck 反应的有效催化剂, 反应后收 率就可达到 95\%, 重复使用 3 次, 产率仍保持在 $83 \%$. 但与聚- $\gamma$-氨丙基硅烷负载的 $\mathrm{Cu}$ 催化剂相比, $\mathrm{Co}$ 催化剂 需要更高的反应温度.

同时, 化学家们对高分子聚合物负载过渡金属型催 化剂催化的 Suzuki 偶联反应做了大量研究. 表 1 列出了 负载型聚合物催化剂主要的应用实例.

1997 年, Jang ${ }^{[24]}$ 首次将聚合物负载钯催化剂应用于 Suzuki 偶联反应，该催化剂催化的卤化物或三氟甲基磺 酸类化合物与有机嗍化合物的偶联反应中表现出较好 的活性, 并且在反应结束后, 催化剂非常容易与反应混 合物分离, 且可重复使用 10 次, 活性未见明显降低(Eq. 6).

2000 年, El-Sayed 等 ${ }^{[25]}$ 报道了聚乙烯吡咯烷酮 (PVP)稳定的纳米钯催化剂, 该催化剂可在水相中实现

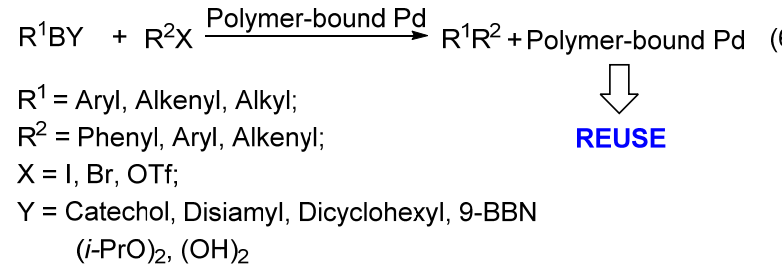

Suzuki 交叉偶联反应(Eq. 7)。碘代芳烃和芳基硼酸在无 配体存在、碱和 $40 \%$ 的乙醇水溶液加热回流的条件下, 可获得较高的产率; 并且催化反应的最初速率与钯浓度 成正比，证明该反应发生在纳米钯的表面. 但是反应结 束后，有金属钯沉淀出现，说明该催化剂的稳定性较差.

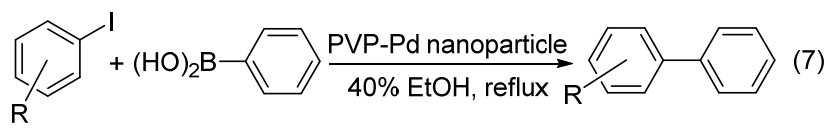

2002 年, Uozumi 等 ${ }^{[26]}$ 报道了一种两亲树脂负载的 钯催化剂的制备及其应用于水相 Suzuki 偶联反应(Eq. 8 ), 采用了 8 种卤代芳烃与 12 种乙烯基和芳基硼酸作底 物, 反应中发现多数底物的反应产率接近定量，而且该 种催化剂在循环使用 3 次后，催化活性未见明显降低。
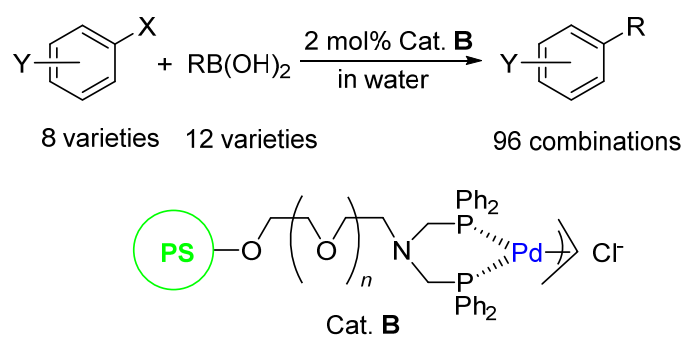

2004 年, $\mathrm{Hu}$ 等 ${ }^{[27]}$ 用一锅法制备了聚 $N, N$-二烷基碳 二酰亚胺负载的纳米钯催化剂 PDHC-Pd, 通过 TEM 可 看出分散的钯纳米颗粒平均尺寸约为 $3 \mathrm{~nm}$ (图 2), 大致 为球形. 研究发现, 无论在传统加热下还是在微波加热 下，这种催化剂对都 Suzuki 偶联反应表现出很高的催化 活性. 在传统加热方式下, 催化剂循环使用 5 次后, 活 性基本保持不变, 产率为 90\%; 然而，在微波加热方式 下，催化剂循环使用 5 次后，其催化活性明显降低(Eq. 9).

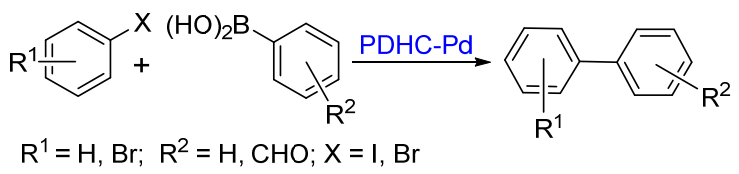

2006 年, Fan 等 ${ }^{[28]}$ 合成并报道了一种高效含膦树枝 状聚合物稳定的钯纳米粒子及其催化 Suzuki 偶联反应 (Scheme 4). 当各种碘代芳烃或溴代芳烃与苯硼酸反应 
表 1 以聚合物为载体型催化剂在 Suzuki 偶联反应中的应用

Table 1 Polymer-supported catalysts in the Suzuki reactions

\begin{tabular}{|c|c|c|c|c|c|c|}
\hline \multicolumn{2}{|c|}{ Catalyst preparation } & \multicolumn{3}{|c|}{ Catalytic reaction } & \multirow{2}{*}{ Yield/\% } & \multirow{2}{*}{ Ref. } \\
\hline Pd source & Support & Aryl halide & Organoborane & Conditions & & \\
\hline $\operatorname{Pd}(0)$ complex & Polymer-bound & (Z)-Styrylbromide & $\begin{array}{l}(E)-\beta \text {-Styryl-1,3,2- } \\
\text { benzodioxaborol }\end{array}$ & $\begin{array}{l}2 \mathrm{~h}, 80{ }^{\circ} \mathrm{C} \text {, benzene, } \\
\mathrm{NaOEt}, 1 \% \mathrm{Pd}\end{array}$ & 96 & {$[24]$} \\
\hline Pd nanoparticles & $\begin{array}{l}\text { Poly(N-vinyl-2- } \\
\text { pyrrolidone) (PVP) }\end{array}$ & Iodobenzene & $\begin{array}{l}\text { 2-Thiopheneboronic } \\
\text { acid }\end{array}$ & $\begin{array}{l}12 \mathrm{~h}, \text { reflux, } 40 \% \\
\text { EtOH, } \mathrm{Na}_{3} \mathrm{PO}_{4} \\
0.3 \% \mathrm{Pd}\end{array}$ & 92 & {$[25]$} \\
\hline$\left[\mathrm{PdCl}\left(\eta^{3}-\mathrm{C}_{3} \mathrm{H}_{5}\right)\right]_{2}$ & Amphiphilic resin & 4-Iodophenol & Phenylboronic acid & $\begin{array}{l}12 \mathrm{~h}, 50{ }^{\circ} \mathrm{C} \text {, water, } \\
\mathrm{K}_{2} \mathrm{CO}_{3}, 2 \% \mathrm{Pd}\end{array}$ & 99 & {$[26]$} \\
\hline Pd nanoparticle & $\begin{array}{l}\text { Poly }(N, N \text {-dihexyl- } \\
\text { carbodiimide })\end{array}$ & $\begin{array}{l}\text { Iodobenzene and } \\
\text { bromobenzene }\end{array}$ & Phenylboronic acid & $\begin{array}{l}0.6 \sim 24 \mathrm{~h}, \text { reflux, } \\
\text { dioxane, } \mathrm{K}_{2} \mathrm{CO}_{3} \text {, } \\
0.5 \% \mathrm{Pd}\end{array}$ & $84 \sim 99$ & {$[27]$} \\
\hline $\mathrm{Pd}(\mathrm{acac})_{2}$ & Phosphine dendrimer & Aryl chlorides & Phenylboronic acid & $\begin{array}{l}48 \mathrm{~h} \text {, reflux, dioxane, } \\
\mathrm{K}_{3} \mathrm{PO}_{4}, 2 \% \mathrm{Pd}\end{array}$ & $51 \sim 94$ & {$[28]$} \\
\hline $\mathrm{Pd}(\mathrm{OAc})_{2}$ & Merrifield resin & Bromobenzene & Arylboronic acid & $\begin{array}{l}4 \text { h, r.t., } \mathrm{EtOH}, \\
\mathrm{K}_{2} \mathrm{CO}_{3}, 0.5 \% \mathrm{Pd}\end{array}$ & $78 \sim 93$ & [29] \\
\hline
\end{tabular}

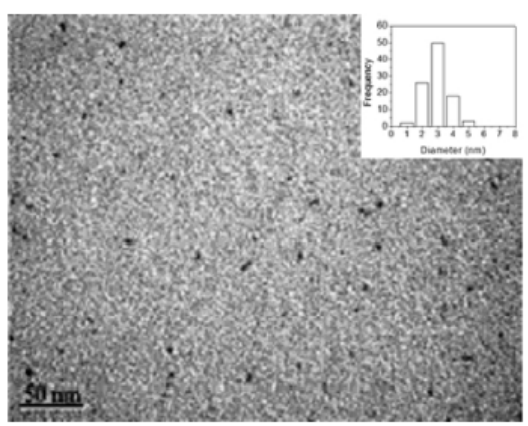

图 2 聚 $N, N$-二烷基碳二酰亚胺负载的纳米钯的 TEM 分析 (bar $=50 \mathrm{~nm}$ )

Figure 2 TEM analysis of palladium nanoparticles stabilized by $\operatorname{poly}(N, N$-dihexylcarbodiimide $)($ bar $=50 \mathrm{~nm})$

时, 仅需要 $0.06 \mathrm{~mol} \%$ 催化剂, 反应即可定量进行; 当各 种氯代芳烃与苯嗍酸反应时, 需要 $2.0 \mathrm{~mol} \%$ 催化剂, 产 率可达 51\% 94\%. 该催化剂在循环使用 8 次后, 仍旧 可保持其活性. 另外, 该催化剂还对氢化反应有明显催 化效果.

Wang 等 ${ }^{[29]}$ 用 Merrifield 树脂作为载体, 经过表面 修饰, 成功将邻菲罗啉单元接枝于树脂表面, 进而负载 过渡金属钯, 制备得到负载的钯催化剂, 然后将其用于 Suzuki 偶联反应, 产率高达 $93 \%$, 需要特别指出的是该 反应在室温下即可进行, 所需催化剂为 $0.50 \mathrm{~mol} \%$, 底 物普适性广, 催化剂的循环使用能力得到了显著提高, 可以重复使用多达 10 次, 催化剂活性保持不变(Eq. 10).

2009 年, Razler 等 ${ }^{[30]}$ 报道了在无配体条件下, 聚乙 二醇 2000 (PEG-2000)负载钯催化的 Suzuki 偶联反应 (Eq. 11). PEG-2000 与钯盐可以快速 $(<1 \mathrm{~min})$ 原位生成 纳米金属钯粒子(图 3), 在 $1.0 \mathrm{~mol} \%$ 的负载钯催化剂下
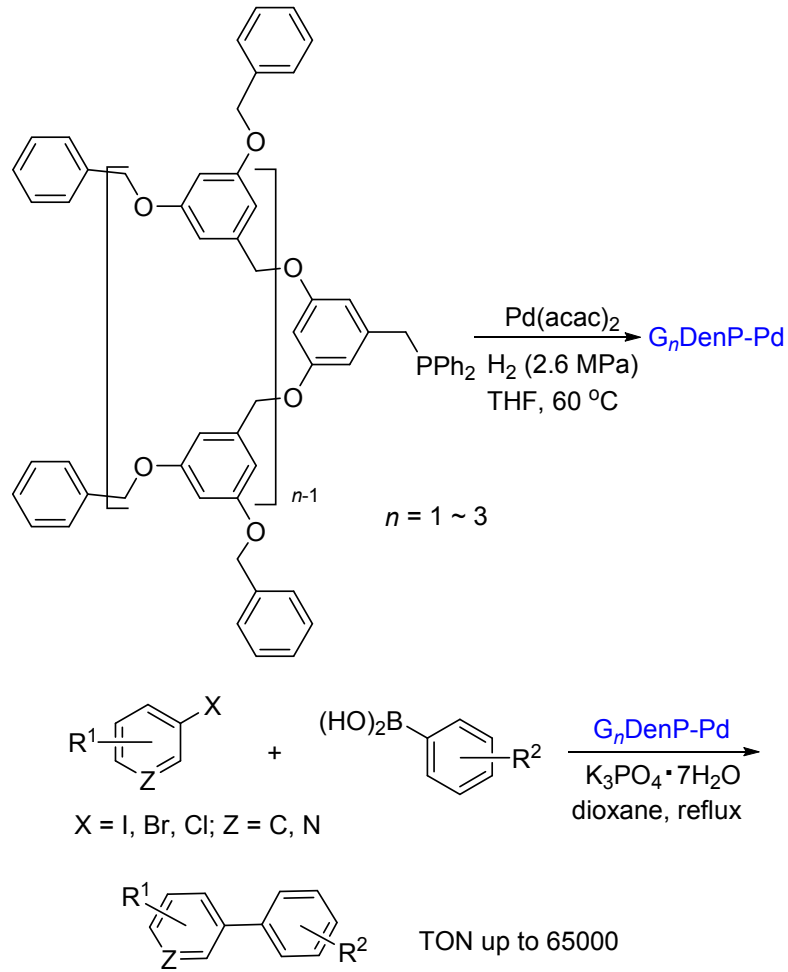

图式 4 树状聚合物-纳米钯催化的 Suzuki 反应 Scheme 4 Polymer-stabilized Pd-nanoparticles for the Suzuki reaction

可较高产率地获得的 Suzuki 交叉偶联目标产物. 在这 里, PEG-2000 既可以用作催化反应的流动相, 又可以动 态的担载催化剂, 通过 “均相反应, 两相分离” 实现均 相催化剂的简单分离.

2009 年, Guo 课题组 ${ }^{[31]}$ 报道了聚 2-氨基硫酚(PATP) 负载纳米金催化的 Suzuki 偶联反应. 该反应以氢氧化钠 为碱，在水相中进行(Eq. 12). 另外值得注意的是，芳基 


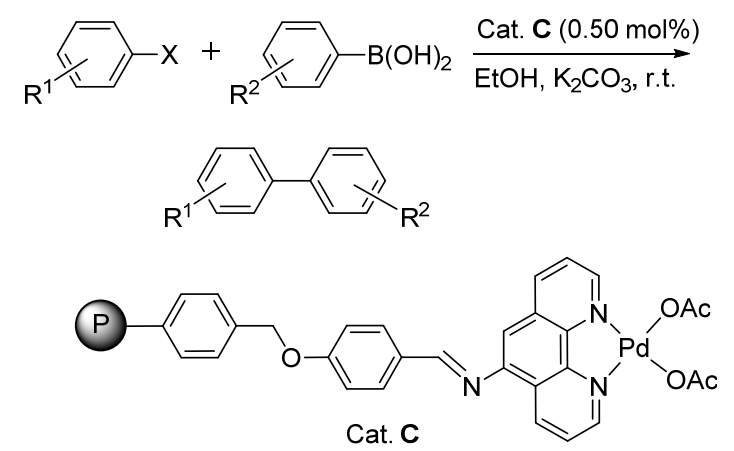

(10)

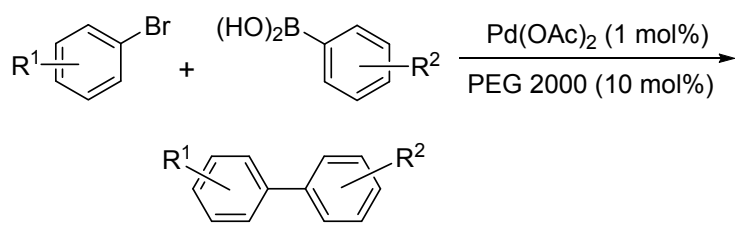

(11)

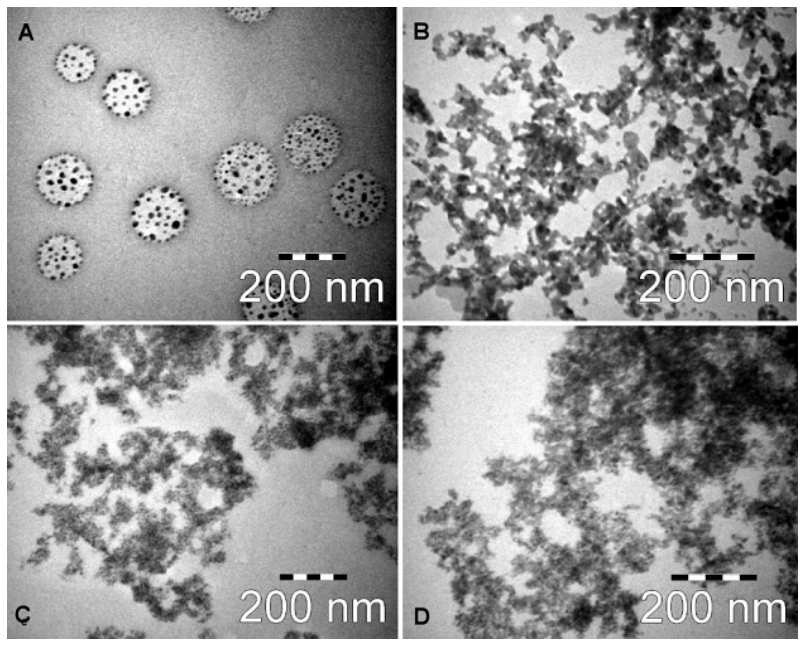

图 3 PEG 2000-Pd 催化剂的 TEM 分析

Figure 3 TEM imaging of the PEG 2000-Pd catalyst After (A) $1 \mathrm{~min}$, (B) $5 \mathrm{~min},(\mathrm{C}) 30 \mathrm{~min}$ and (D) $2 \mathrm{~h}$

氯化物与苯嗍酸反应的产率几乎和相应的溴化物或碘 化物与苯硼酸反应的产率相当.

2010 年, Wang 等 ${ }^{[32]}$ 报道了一种高效的聚苯乙烯负 载钯催化剂催化的芳基溴或芳基碘与芳基铋合物的交 叉偶联反应, 在良好的收率同时, 官能团的忍耐性也较 好(Eq. 13). 该聚合催化剂可从反应混合物中方便地分 离, 重复使用 10 次, 没有任何明显的催化活性降低.

Wang 小组 ${ }^{[33]}$ 继金属亚微米镍粉在异丙醇中催化末 端炔烃与卤代(碘或澳)芳烃的偶联反应的研究后, 制备 了一种可循环使用的聚乙烯吡咯烷酮(PVP)负载纳米钯 催化剂 ${ }^{[34]}$. 该催化剂可在无配体、无铜、无有机胺的条 件下, 实现催化碘代或溴代芳烃与末端炔烃的 Sonogashira 偶联反应, 产率高达 $98 \%$, PVP 负载的纳米钯催化 剂反应后经过简单处理, 可重复使用 8 次, 活性不变 (Eq. 14).

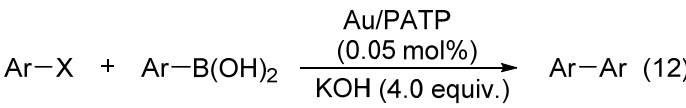

$$
\begin{aligned}
& \mathrm{H}_{2} \mathrm{O}, 4 \mathrm{~h}
\end{aligned}
$$

Aryl halide $\quad$ Arylboronic acid 1
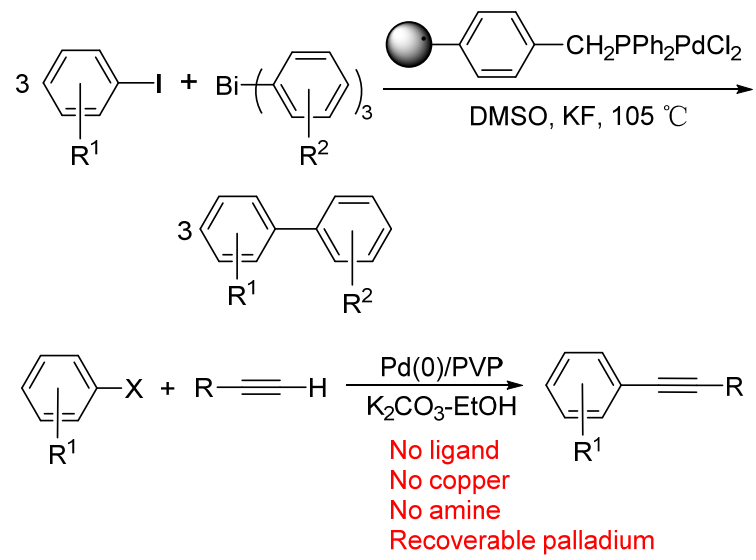

\section{2 离子液体负载过渡金属催化剂}

离子液体是一种在室温或者低于 $100{ }^{\circ} \mathrm{C}$ 时呈液态 的物质，一般由无机阴离子和有机阳离子组合而成. 在 过去的十几年中，离子液体曾受有机化学家的广泛关 注, 开始人们仅将离子液体当作绿色溶剂使用, 近年来 离子液体用作催化剂载体亦有报道, 事实上功能化离子 液体就是一类很好的过渡金属催化剂载体.

2003 年, Audic 等 ${ }^{[35]}$ 通过多步合成得到烷基咪唑盐 负载的 Grubbs 钉催化剂(Eq. 15), 并用于烯烃复分解反 应. 实验结果表明, 烷基咪唑盐负载的 Grubbs 催化剂催 化活性高, 反应产率在 $95 \%$ 以上, 并且催化剂多次循环 使用而不失活性，在第 10 次循环使用后产率仍保持在 95\%.

2004 年, Corma 和 García 等 ${ }^{[36]}$ 报道了肟配位的钯催 化剂, 再通过引入链状咪唑盐制备了离子液体催化剂, 随后试验了这种钯配合物用于 Heck 和 Suzuki 交叉偶联 反应的催化活性(Scheme 5). 


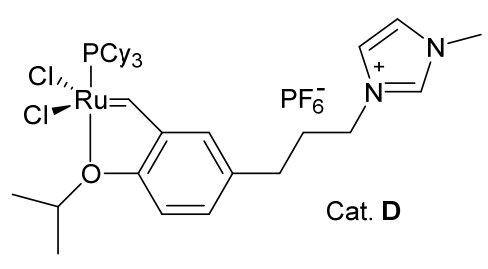

$\underset{60{ }^{\circ} \mathrm{C}, 45 \mathrm{~min}}{\stackrel{(2.5 \mathrm{~mol} \%)}{\longrightarrow}}$<smiles>CC(=O)c1ccc(O)cc1</smiles><smiles></smiles><smiles>[2H]C(CC)CCCCCn1cc[n+]([B-])c1</smiles><smiles>[X]c1ccccc1</smiles>
$\mathrm{X}=\mathrm{Cl}, \mathrm{Br}, \mathrm{I}$<smiles>O=Cc1ccccc1</smiles>

图式 5 离子液负载的钯催化的 Heck 和 Suzuki 反应 Scheme 5 Ionic liquid supported Pd for the Heck and Suzuki reactions

2004 年, Dyson 等 ${ }^{\left[{ }^{[7]}\right.}$ 报道了系列腈基官能化的离子 液体 1 4 配位的钯催化剂 $5 \sim 9$ 及在 Suzuki 和 Stille 偶 联反应中的应用. 研究表明, 所有的钯配合物均显示出 良好的催化活性(Scheme 6).

2006年, Shreeve 等 ${ }^{[38]}$ 制备了一系列吡唑和 3,5-二甲 基吡唑官能化氮配位的室温离子液体及其配位的钯催 化剂(Scheme 7). 随后研究了该载体催化剂在 Heck, Suzuki 及 Sonogashira 偶联反应中的应用.

2007 年, 该课题组 ${ }^{[39]}$ 又制备了 (2-吡啶基)-咪唑的 离子液体配位的钯催化剂, 并研究了其催化的 Heck 偶 联反应, 此类钯催化剂在 Heck 反应中表现出了非常好 的稳定性与循环回收再使用性(Scheme 8).

2006 年, Wang 课题组 ${ }^{[40]}$ 将功能化离子液体作为一 种高效可循环的反应介质, 在无膦配体的条件下, 醋酸 钯催化的 Heck 反应(Eq. 16). 该反应使用 $[\mathrm{HEmim}]\left[\mathrm{BF}_{4}\right]$ 作为溶剂, 磷酸钾作为碱, 乙酸钯作为催化剂. 钯和离 子液体可以回收并循环使用, 连续 6 次实验, 发现催化
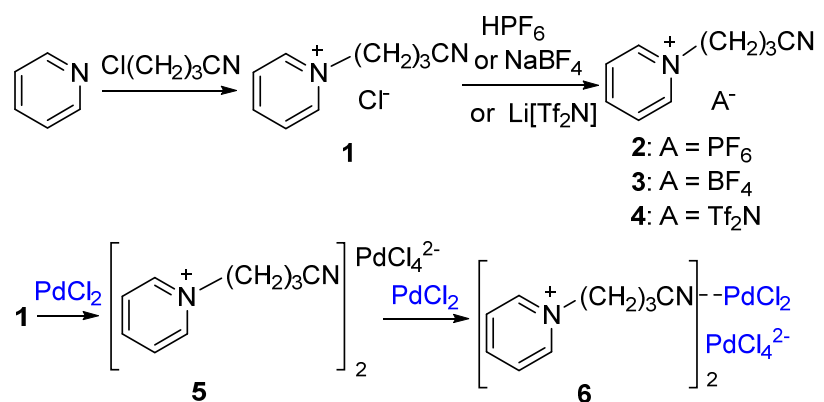

$$
2 \sim 4 \stackrel{\mathrm{PdCl}_{2}}{\longrightarrow}\left[\begin{array}{l}
2 \mathrm{~A}^{-} \\
\text {7: } \mathrm{A}=\mathrm{PF}_{6} \\
\text { 8: } \mathrm{A}=\mathrm{BF}_{4} \\
\text { 9: } \mathrm{A}=\mathrm{Tf}_{2} \mathrm{~N}
\end{array}\right.
$$

图式 6 腈基官能化离子液负载的钯催化剂

Scheme 6 Nitrile functionalized ionic liquid coordinated Pd catalyst
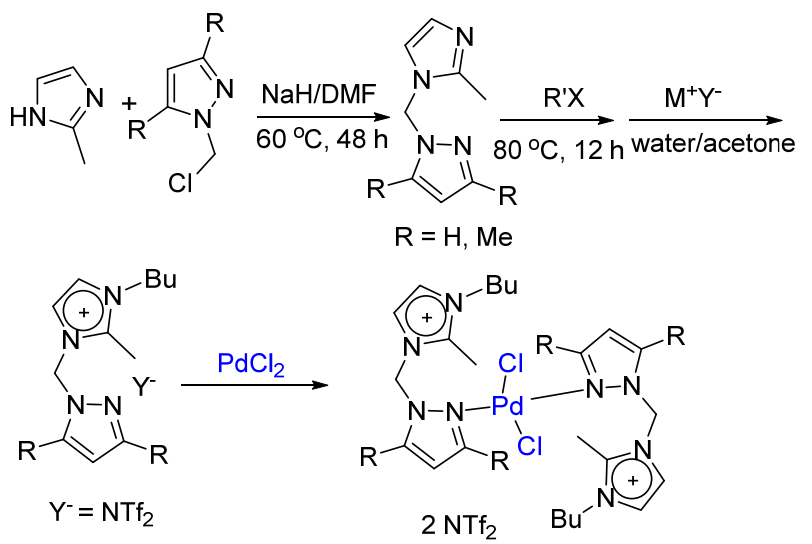

图式7 吡唑官能化离子液配位的钯催化剂

Scheme 7 Pyrazolyl-functionalized ionic liquids and their palladium(II) complexes
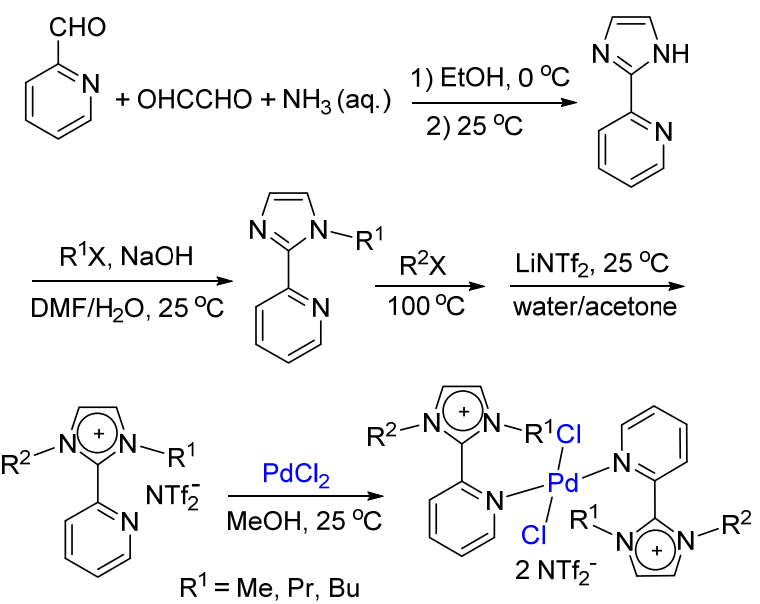

图式 8 吡啶官能化离子液配位的钯催化剂

Scheme 8 Pyridyl-functionalized imidazolium-based ionic liquid and its palladium(II) complex 
剂活性无显著降低. 该课题组 ${ }^{[41]}$ 后来发现该反应在三 乙醇胺中也可以进行.

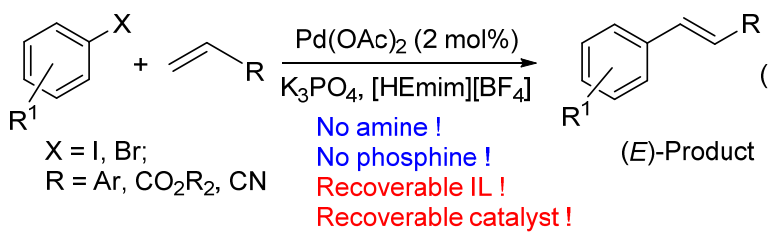

2009 年, Lombardo 等 ${ }^{[42]}$ 通过使用预制的、在空气中 可稳定存在的、容易处理的含膦配体的离子液体钯配合 物，实验了其在 Suzuki 偶联反应中的应用(Scheme 9). 在较短的反应时间和较温和的条件下, 催化剂循环使用 6 次后, 没有显示出催化剂的结构破坏亦没有显著的催 化活性损失

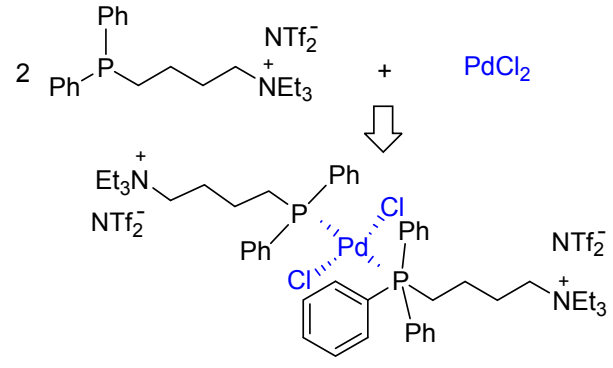

cat. Pd

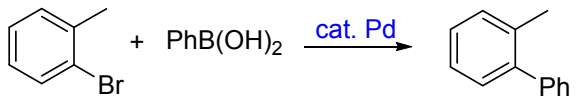

图式 9 含膦配位的钯催化剂用于 Suzuki 反应

Scheme 9 Diphenylphosphine palladium complex for the Suzuki reaction

同年, Wang 小组 ${ }^{[43]}$ 以三正丁胺、二乙醇胺及 $1,4-$ 二 溴丁烷为原料制备了离子液体(Scheme 10). 该功能化 离子液体含有氨基单元, 即具有碱的特征. 随后以 Heck 反应为模板, 考察了功能化离子液体在该反应中的表 现. 结果表明其性能优于普通离子液体, 主要体现在功 能化离子液体中无需额外加入碱, 就能顺利实现过渡金 属钯催化的 Heck 反应, 而且反应底物范围广, 产率高, 该离子液体可循环使用 6 次, 产物收率基本保持不变.

2011 年, Wang 等 ${ }^{[44]}$ 报道了(2-吡啶基)-咪唑的离子 液体配位的钯催化剂在水相中的 Suzuki 偶联反应，无论 芳基溴化物乃至化学性质惰性的芳基氯都能发生交叉 偶联反应, 并且该催化剂极其稳定, 即使放置于空气中 长达数月，也不发生团聚(Scheme 11).

\section{4 有机一无机杂化材料载体负载的过渡金属催 化剂}

通常无机材料如二氧化硅价廉易得, 且具有比有机 材料更好的机械和热稳定性. 若将有机配体负载到无机

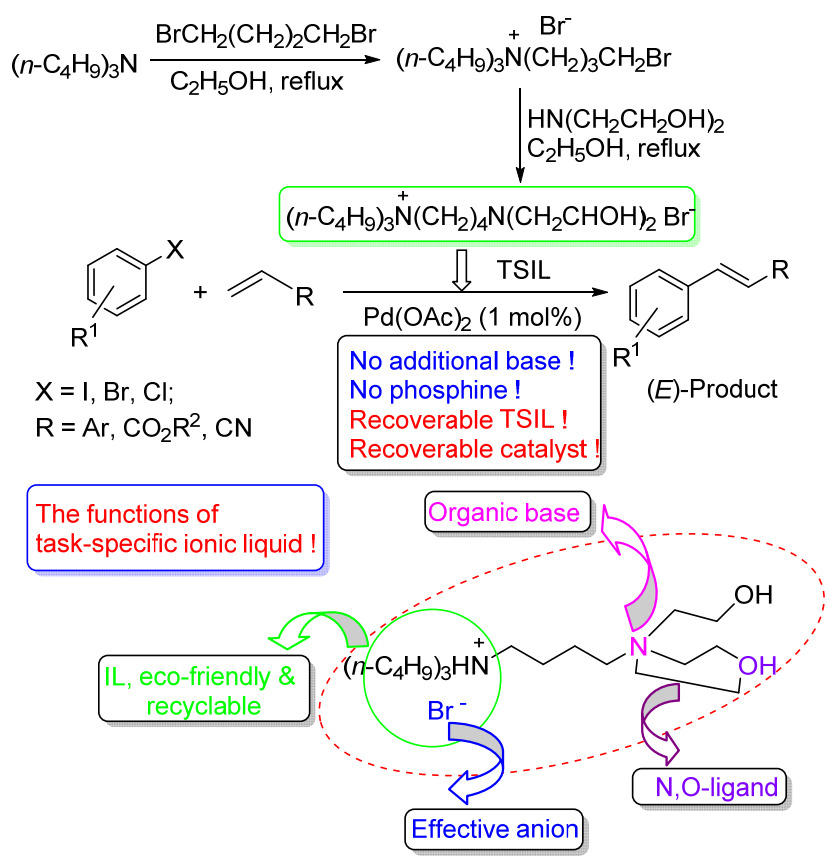

图式 10 多功能化离子液体用于 Heck 反应

Scheme 10 Multi-functionalization ionic liquid and its application in Heck reaction

材料表面，则既可提高催化剂的表面积，又可降低成本. 有机一无机杂化载体就是指对无定型硅胶、MCM-41、

SBA-15 等无机材料的表面或空腔进行修饰，通过化学 反应在其表面或空腔嫁接上含有特定官能团的有机分 子的一类复合材料. 然后通过配位螯合的方式将金属催 化剂配位于功能基团上，从而实现催化剂的负载.

2006 年, Wang 课题组 ${ }^{[45]}$ 研究了以无机多孔性材料 硅胶表面羟基经过含乙二胺单元有机硅氧烷试剂接枝 修饰制备的有机一无机杂化材料为载体，通过接枝含氮 配体的螯合作用引入 $\operatorname{Pd}(\mathrm{II})$ ，制得有机一无机杂化材料负 载的高活性钯催化剂, 并且成功用于催化 Sonogashira 反应. 该反应在无铜、无有机碱及膦配体的情况下即可 完成，反应在环境比较友好的溶剂乙醇中回流 $6 \mathrm{~h}$, 产 物最高收率达 $98 \%$ ，卤代芳烃可以扩大到溴代物，且催 化剂可反复应用 30 次不失活性(Scheme 12). 研究发现, 该催化剂亦可催化 Suzuki 反应(Eq. 17) ${ }^{[46]}$. 同年他们报 道了该载体材料负载 $\mathrm{Cu}(\mathrm{I})$ 催化的 Sonogashira 偶联反

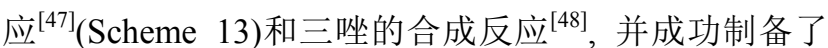
该配体负载 $\mathrm{Cu}(\mathrm{II})$ 催化剂，用于催化 Ullmann 醚化反 应 $^{[49]}$.

2008 年，该小组 ${ }^{[50]}$ 报道了一种新颖的二氧化硅负 载的脯氨酸铜(I)的有机-无机杂化材料作为一种高效催 化剂用于 Sonogashira 反应，末端炔烃在该催化剂下与 芳基碘化物和溴化物顺利反应得到相应的交叉偶联产 物，收率良好，催化剂可通过对反应液的简单过滤回收， 并连续循环使用 6 次催化活性无明显降低(Eq. 18). 

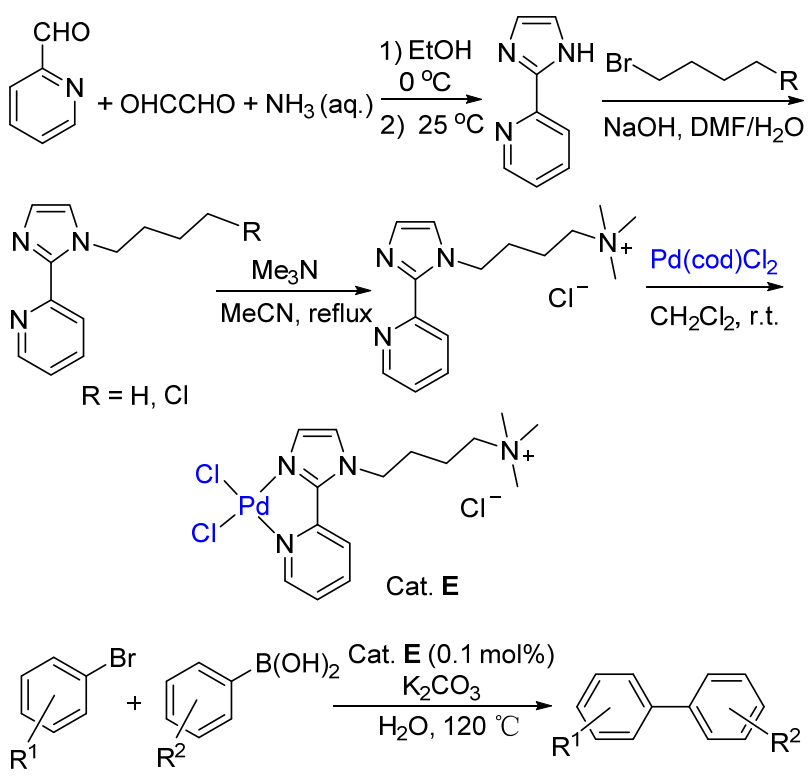

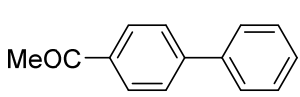

$97 \%$

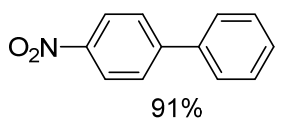

$91 \%$

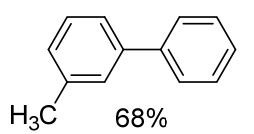

$\mathrm{MeOC}$

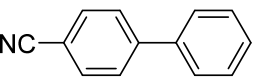

$99 \%$
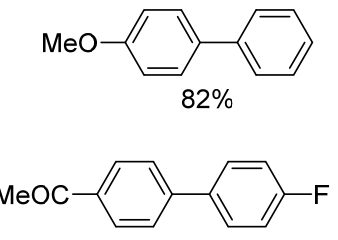

$92 \%$

图式 11 离子型含氮配位的钯催化剂用于 Suzuki 反应

Scheme 11 Pd chelating complex of ionic nitrogen-containing ligand for the Suzuki reaction
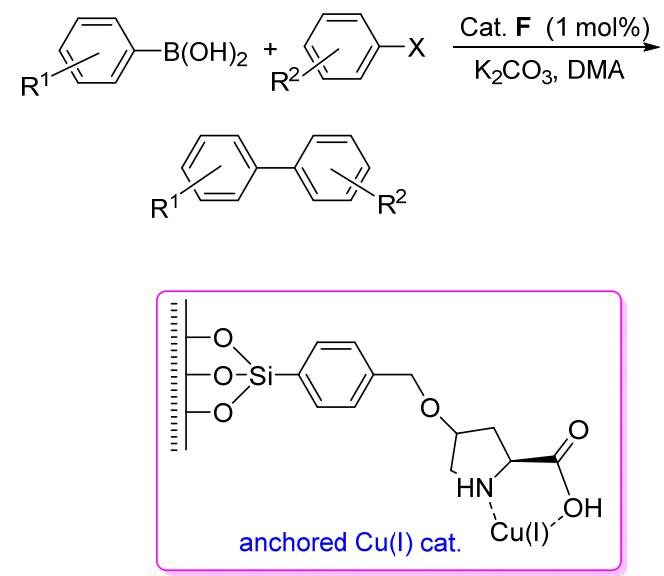

$\mathrm{R}^{1}-\mathrm{X}+\mathrm{R}^{2} \equiv \mathrm{H} \underset{\mathrm{K}_{2} \mathrm{CO}_{3}, \mathrm{DMF}}{\stackrel{\text { anchored } \mathrm{Cu}(\mathrm{l}) \text { cat. }}{\longrightarrow}} \mathrm{R}^{1}=\mathrm{R}^{2}$

同年他们 ${ }^{[51]}$ 还报道了经吡啶官能团修饰的二氧化 硅载体负载钯催化剂, 并将其应用于催化 Heck 反应(Eq. 19). 用该负载催化剂, 实现在无膦、碳酸钾为碱、 $N, N$ 二甲基甲酰胺为溶剂的条件下碘苯、溴苯和氯苯与苯乙

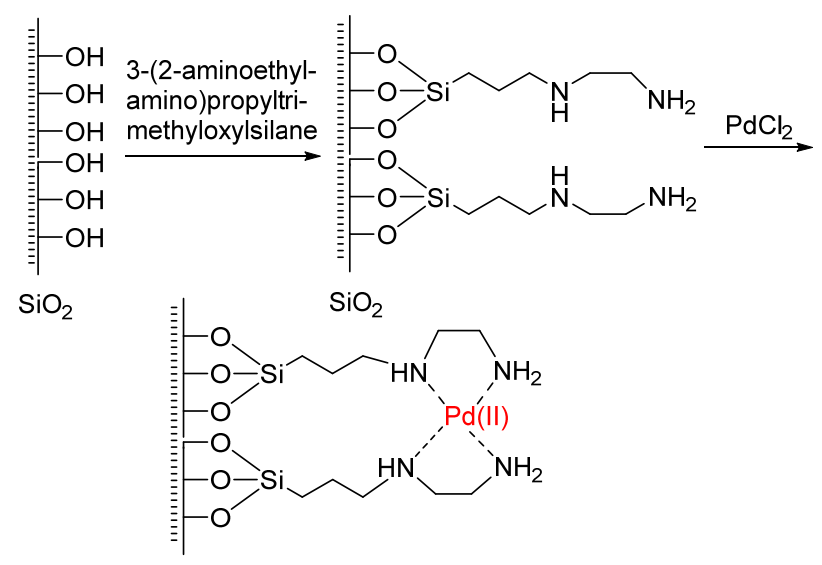

$\mathrm{SiO}_{2} \quad$ Cat. $\mathbf{F}$

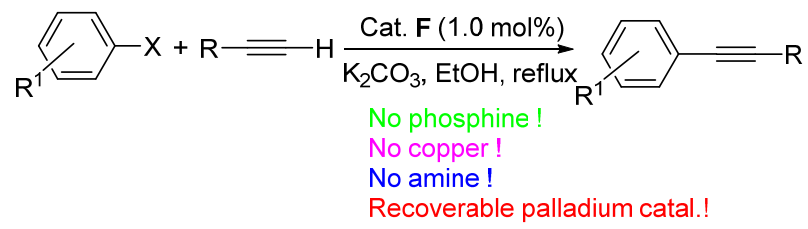

图式 12 有机一无机杂化材料负载钯催化的 Sonogashira 反应 Scheme 12 Sonogashira reaction catalyzed by immobilization of Pd in organic-organic hybrid materials

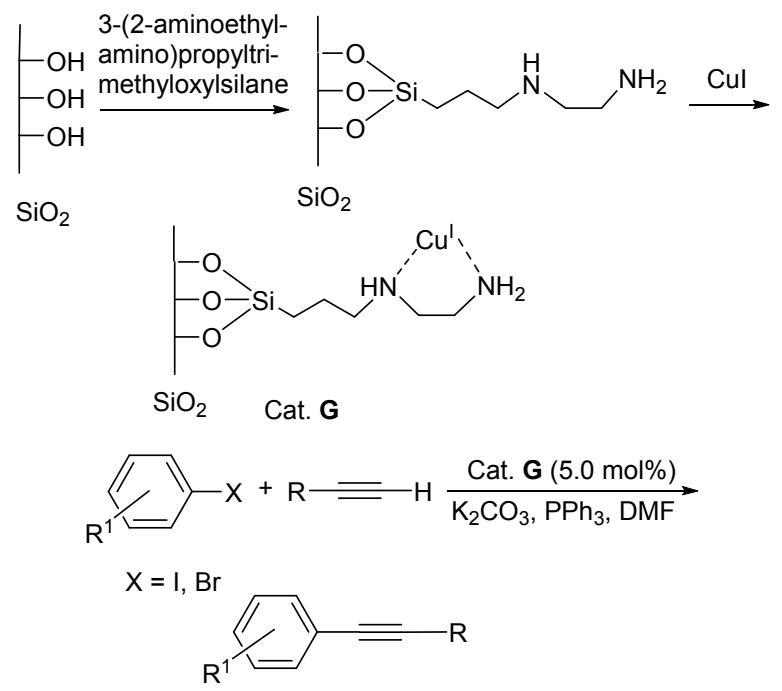

图式 13 有机-无机杂化材料负载铜催化的 Sonogashira 反应 Scheme 13 Sonogashira reaction catalyzed by immobilization of $\mathrm{Cu}$ in organic-inorganic hybrid materials

烯的偶联反应，产率优良. 同时该小组 ${ }^{[52]}$ 报道了以表面 经过氯化茮接枝修饰的无机多孔性材料硅胶为载体，负 载氮杂环卡宾铜配合物以及碘化亚铜以制备负载的高 活性铜催化剂(Scheme 14), 成功用于催化端基炔、醛、 胺三分子偶联反应. 对芳香醛、脂肪醛，芳基炔、脂肪 炔, 芳香胺、脂肪胺都有良好的催化效果. 催化剂 $\mathbf{H}$ 催 化该反应可在室温及无溶剂条件下进行, 催化剂 I 催化 该反应需要加热至 $80{ }^{\circ} \mathrm{C}$. 这两种催化剂均可多次循环 使用而不失活性, 符合绿色化学要求. 其中催化剂 $\mathbf{H}$ 亦 
可用于催化炔与叠氮化合物的 $[3+2]$ 环加成反应 ${ }^{[53]}$.

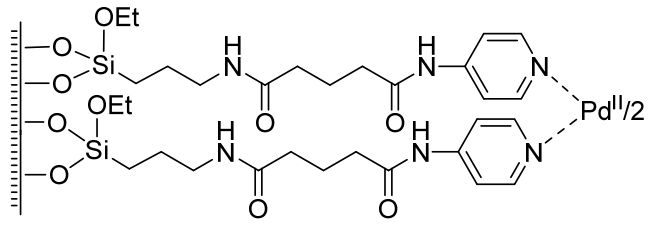

Cat. G

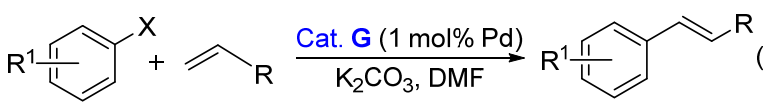

$\mathrm{X}=\mathrm{I}, \mathrm{Br}, \mathrm{Cl} ; \mathrm{R}=\mathrm{Ph}, \mathrm{CO}_{2} \mathrm{R}^{2}$

(E)-Product
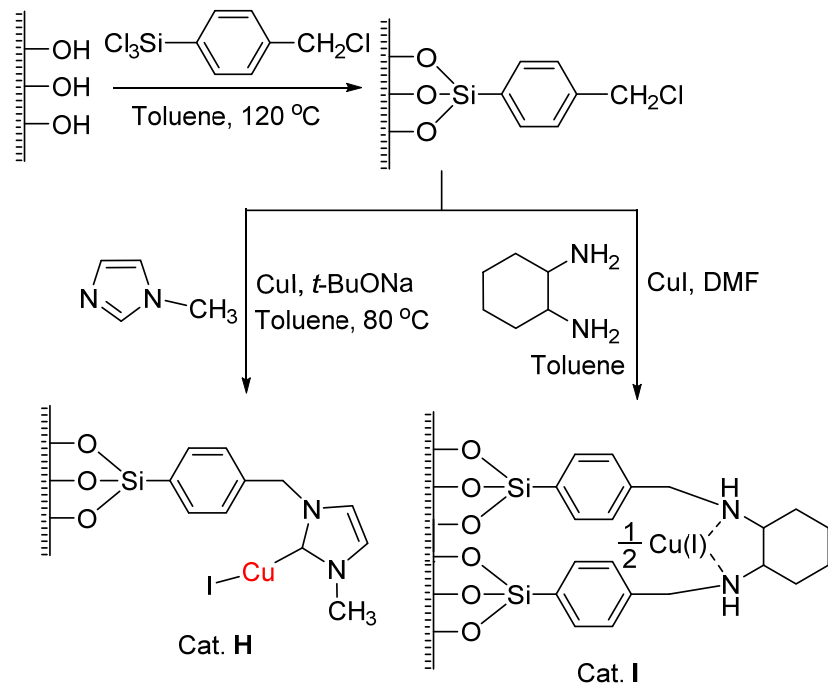

图式 14 有机-无机杂化材料负载铜催化剂

Scheme 14 Immobilization of copper in organic-inorganic hybrid materials

2011 年, Wang 小组 ${ }^{[54]}$ 设计并制备了表面有机膦官 能化的硅胶, 继而制备了硅胶负载的有机膦-钯配合物, 并将其应用于 Suzuki 偶联反应，取得了很好的反应效 果, 对碘代烃、溴代烃和氯代烃都有良好的催化效果 (Scheme 15). 实验结果表明, 该催化剂催化效率高, 底 物选择广谱性好, 并且催化剂可多次循环使用而不失活 性, 符合绿色化学要求. 他们设计并制备了系列硅胶负 载的钯、铜催化剂, 并成功用于催化 Suzuki, Sonogashira, Heck 等有机反应, 催化剂催化效率高, 可多次循 环使用而不降低活性，具有潜在的工业应用前景.

Cai 小组在 MCM-41 负载方面做了大量工作. 2007 年, Cai 等 ${ }^{[55]}$ 报道了 MCM-41 负载双齿膦钯(0)催化剂, 它是一种高活性可循环使用的催化剂, 特别是对芳基碘 化物的 Sonogashira 反应极为有效. 2013 年, Cai 等 ${ }^{[56]}$ 报 道了一种快速高效的二氧化硅材料无膦配体的聚合 MCM-41 负载的钯催化剂, 可以高产率的催化酰氯和芳 基铋合物的交叉偶联反应. 该催化剂通过简单的过滤回 收, 循环使用 10 次而催化活性没有明显的下降(Eq. 20).

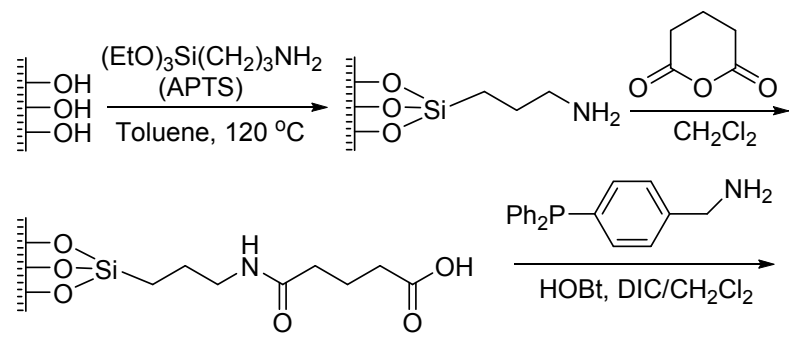

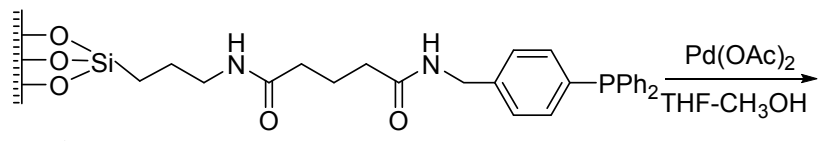<smiles>CCOP(C)c1ccc(-c2ccccc2)cc1</smiles>
Supported palladium catalyst

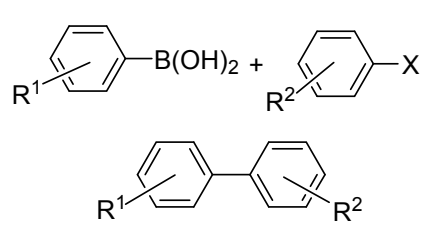

Supported palladium catalyst ( $0.50 \mathrm{~mol} \%)$

$\mathrm{MeOH} / \mathrm{H}_{2} \mathrm{O}(\mathrm{V} / \mathrm{V}=1 / 1)$ Base, r.t.

图式 15 有机-无机杂化材料负载钯催化剂用于 Suzuki 反应 Scheme 15 Immobilization of Pd in organic-inorganic hybrid materials for the Suzuki reaction

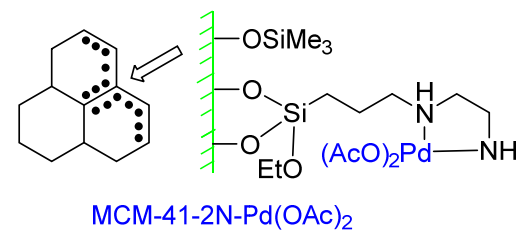

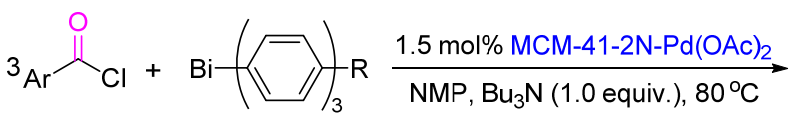<smiles>[R]c1ccc(C(=O)[AlH2])cc1</smiles>

\section{5 磁性纳米粒子负载的过渡金属催化剂}

近几年来发展的将二氧化硅材料嫁接在磁性纳米 颗粒铁的氧化物上，实现了循环回收的高效性与简便 性. 由于纳米粒子的尺寸在纳米级别, 使得纳米粒子催 化体系相比于传统的催化体系具有较好的优越性. 由于 尺寸的减小, 表面积与体积之比增加, 使得催化剂的活 性成分的有效表面积增大，在很大程度上增加了反应物 分子与催化剂之间的接触. 这种增强的相互作用极大地 促进了非均相催化，提高了反应速率，使之催化活性在 一定程度上接近于均相催化. 这种催化剂不溶于水且具 有磁性，可以通过磁铁使催化剂简单而有效地从反应混 
合物中分离, 提高了磁性纳米粒子催化剂的再利用 性(图 4) ${ }^{[57]}$.

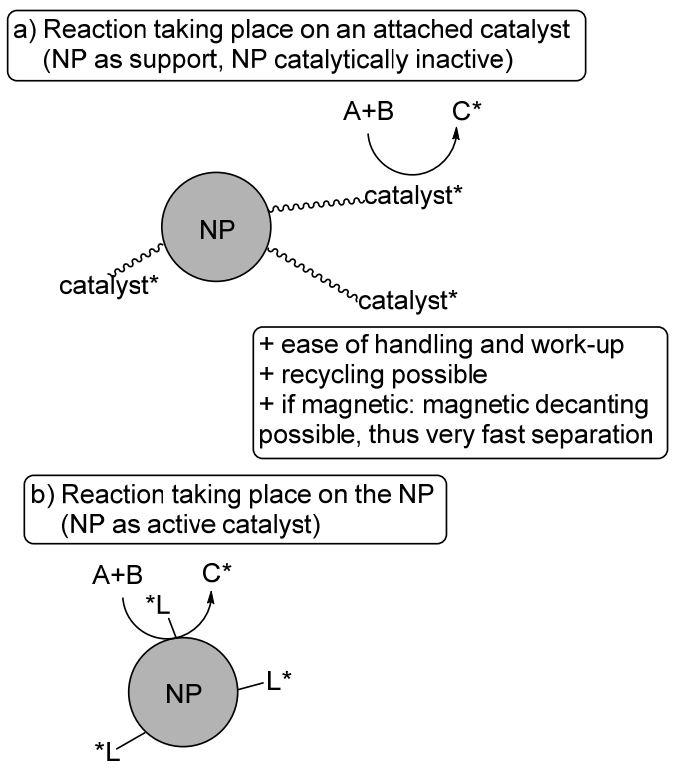

图 4 磁性纳米粒子负载催化剂策略

Figure 4 The design of magnetic nanoparticle immobilized catalyst

2006 年, Gao 研究小组 ${ }^{[58]}$ 将合成的 $N$-杂环卡宾钯 $(\mathrm{Pd}-\mathrm{NHC})$ 连接到油酸表面, 然后固定在稳定的水溶性 纳米 $\gamma-\mathrm{Fe}_{2} \mathrm{O}_{3}$ 上, 合成了一个稳定、高活性的磁性纳米粒 子催化剂(Eq. 21). 该催化剂在 Suzuki 偶联反应中表现 出很高的催化活性, 无论是带有吸电子基团的还是带有 供电子基团的碘苯、溴苯和芳基嗍酸反应，几乎都能得 到定量的产率.
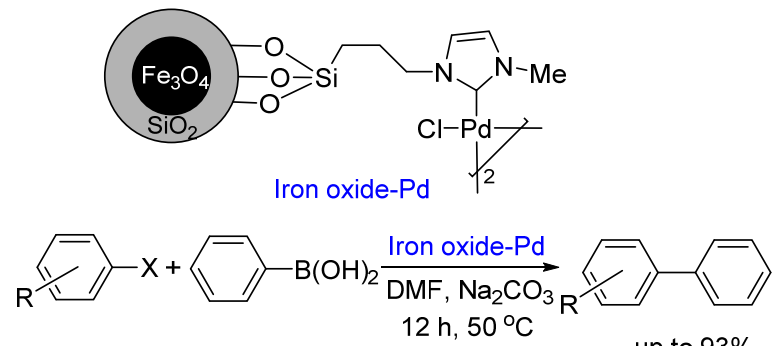

$\mathrm{R}=\mathrm{Me}, \mathrm{OMe}, \mathrm{OAc} ; \mathrm{X}=\mathrm{I}, \mathrm{Br}$ $12 \mathrm{~h}, 50^{\circ} \mathrm{C}$

2007 年, Manorama 研究小组 ${ }^{[59]}$ 合成了 $\mathrm{NiFe}_{2} \mathrm{O}_{4}$ 的多 巴胺(DA)-Pd 催化剂, 并且在催化苯乙烯与芳基碘化物 和溴化物的 Heck 偶联反应中显示出良好的转化率和选 择性, 反应条件温和(Eq. 22), 甚至对于氯代芳烃, 也得 到理想的反应效果.

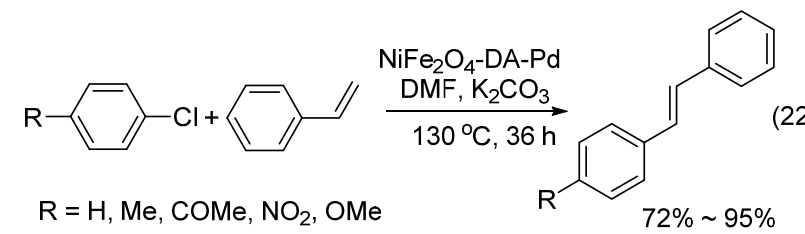

2010 年, Lee 研究小组 ${ }^{[60]}$ 报道了一种活性特别高的 磁性纳米粒子催化剂. 他们首先设计合成了一种新的 $\mathrm{Pd}$ 的配合物, 然后在回流的甲苯中通过三乙氧基硅烷 官能团将其连接到磁性纳米粒子上 (Scheme 16). 以 $\mathrm{K}_{2} \mathrm{CO}_{3}$ 为碱, 水为溶剂, 在 $\mathrm{TBAB}$ 作相转移剂的条件下, 可以很好的催化芳基氯化物与苯嗍酸的偶联反应.

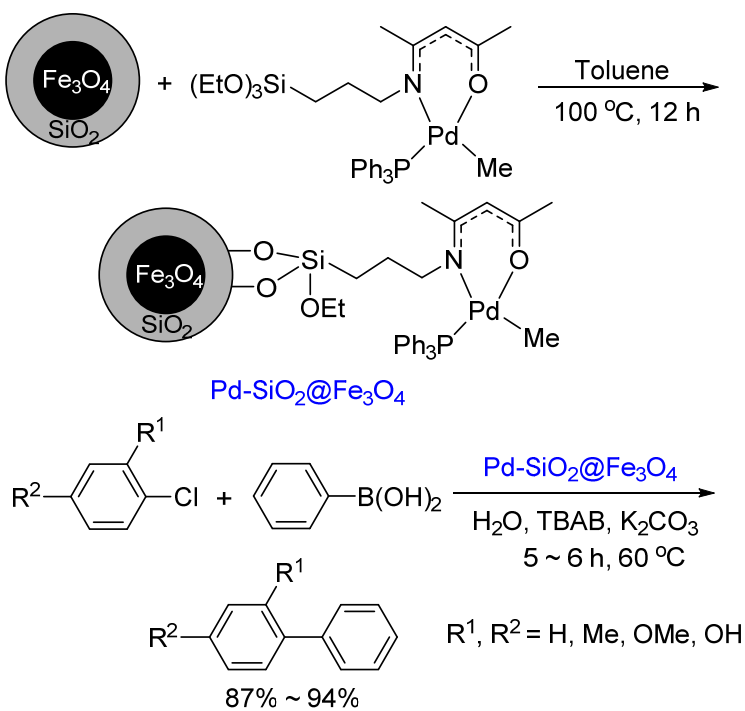

图式 $16 \mathrm{Fe}_{3} \mathrm{O}_{4} @ \mathrm{SiO}_{2}-\mathrm{Pd}$ 催化的 Suzuki 反应 Scheme $16 \quad \mathrm{Fe}_{3} \mathrm{O}_{4} @ \mathrm{SiO}_{2}$-Pd catalyst for the Suzuki reaction

2012 年, Wang 课题组 ${ }^{[61]}$ 报道了磁性纳米粒子负载 钯催化的 Suzuki, Sonogashira 和 Heck 反应, 催化剂的磁 分离性能非常优越，可以回收再利用至少 8 次无显著损 失其催化活性(Scheme 17). 研究还发现这一催化剂同 样可以应用在各种烷氧基芳基硅试剂与卤代芳基化合 物的 Hiyama 交叉偶联反应上，取得了非常好的效果 ${ }^{[62]}$, 简洁的操作方法以及良好的回收效率，使其成为 Hiyama 反应理想的催化体系.

2013 年, Luo 等 ${ }^{[63]}$ 报道了一种 Click 产物为配体的 磁性纳米颗粒负载钯催化剂在乙醇/水溶液中的 Suzuki 偶联反应(Scheme 18). 该体系钯的用量低，无需膦配 体，并且该催化剂可以很容易地从反应体系分离再利 用，循环使用多次而无显著的活性降低.

2013 年, Zolfigol 等 ${ }^{[64]}$ 报道了稳定的磁性钯纳米催 化剂催化的芳基酚化反应和 Sonogashira 交叉偶联反应 (Scheme 19). 反应体系使用 $\mathrm{NaOH} /$ 水中，非常简单和高 效的实现了偶联反应，并且催化剂的回收利用 6 次而不 降低活性(图 4).

2014 年, Sardarian 等 ${ }^{[65]}$ 报道了一种席夫碱配合物的 官能化磁性纳米颗粒负载钯催化剂在 Sonogashira 反应 中的应用, 包括活性较低的氯代芳烃也能较好地进行反 应(Scheme 20), 该体系钯的用量低，无须膦配体，并且 


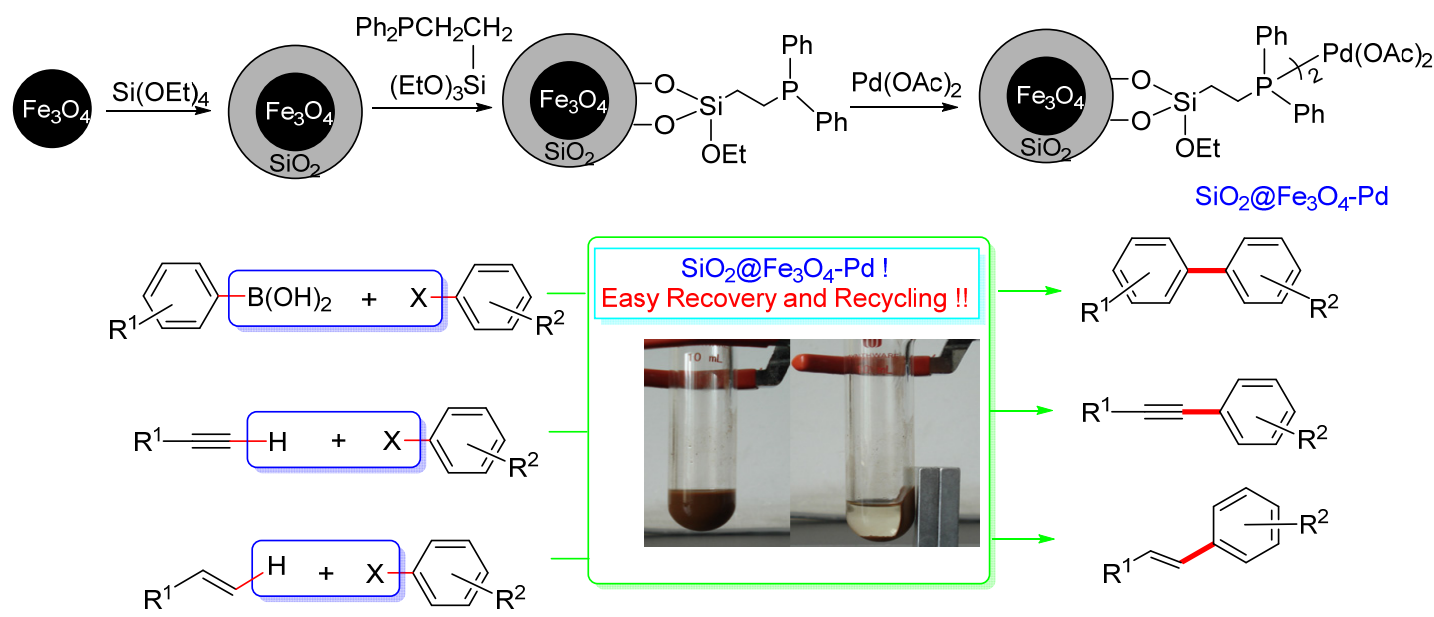

图式 $17 \mathrm{SiO}_{2} @ \mathrm{Fe}_{3} \mathrm{O}_{4}-\mathrm{Pd}$ 催化剂的制备及其在 Suzuki, Sonogashira 和 Heck 反应中的应用

Scheme 17 Preparation of $\mathrm{SiO}_{2} @ \mathrm{Fe}_{3} \mathrm{O}_{4}-\mathrm{Pd}$ catalyst and its application in Sukuzi, Sonogashira and Heck reactions

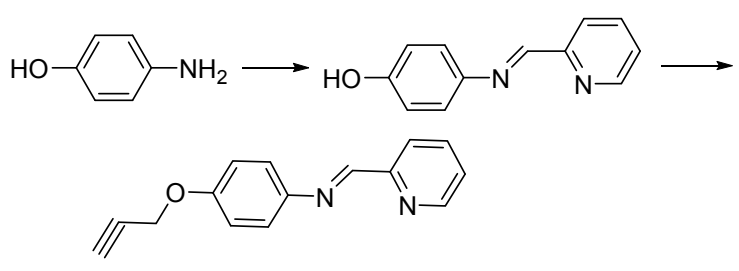

$(\mathrm{EtO})_{3} \mathrm{Si} \frown \mathrm{Cl}_{(\mathrm{EtO}){ }_{3} \mathrm{Si}} \longrightarrow \mathrm{N}_{3} \longrightarrow$

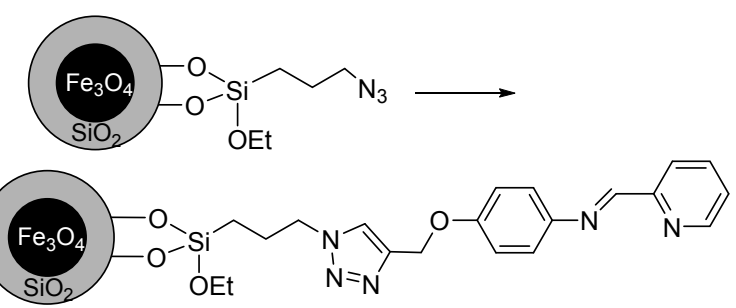

$\stackrel{\mathrm{Pd}(\mathrm{II})}{\longrightarrow} P d_{\mathrm{np}} @ \mathrm{MNP}$

图式 18 磁性纳米粒子负载钯催化剂的制备

Scheme 18 Preparation of magnetic nanoparticle immobilized Pd catalyst

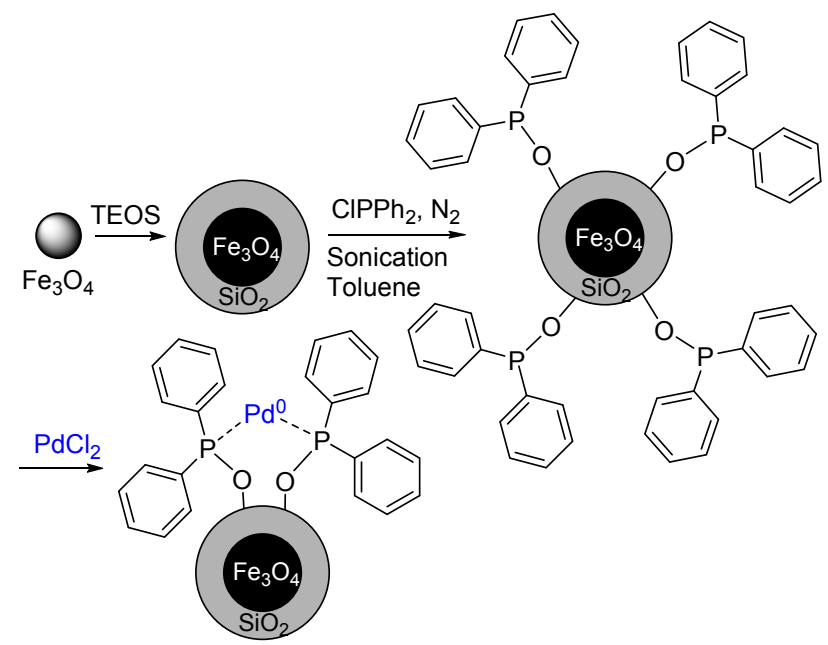

图式 19 磁性纳米粒子负载钯催化剂

Scheme 19 Magnetic nanoparticle immobilized Pd catalyst

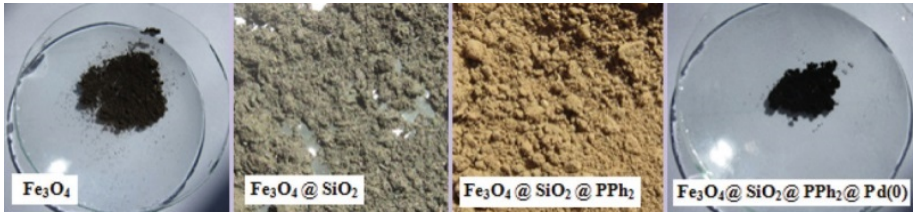

图 5 磁性分散体系的分布制备

Figure 5 Preparation of the magnetically separable system

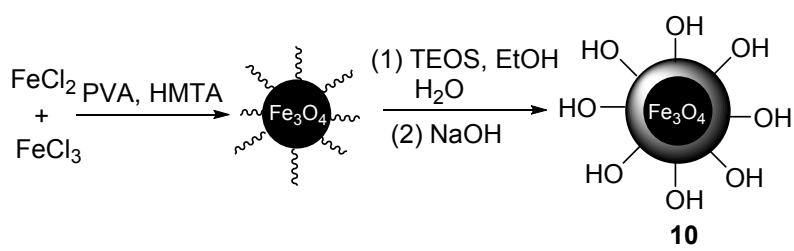

$(\mathrm{EtO})_{3} \mathrm{SiCH}_{2} \mathrm{CH}_{2} \mathrm{CH}_{2} \mathrm{NH}_{2} \underset{\text { (1) Salicylaldehyde, EtOH, r.t., } 6 \mathrm{~h}}{(\text { Pd(OAc) })_{2}}$<smiles>CCOCCCN1Cc2ccccc2O1</smiles>

(EtO) ${ }_{3} \mathrm{SiCH}_{2} \mathrm{CH}_{2} \mathrm{CH}_{2} \mathrm{~N}^{\prime} \mathrm{O}$
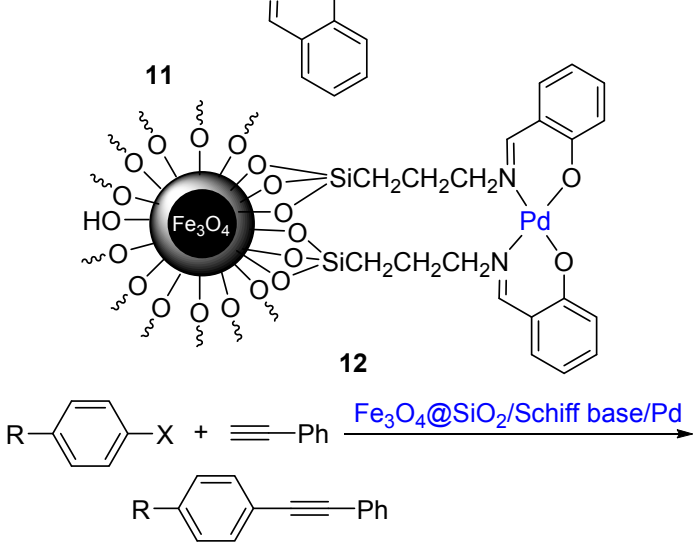

图式 $20 \quad \mathrm{Fe}_{3} \mathrm{O}_{4} @ \mathrm{SiO}_{2} / \mathrm{Pd}$ 催化 Sonogashira 反应

Scheme $20 \quad \mathrm{Fe}_{3} \mathrm{O}_{4} @ \mathrm{SiO}_{2} / \mathrm{Pd}$ catalyst for Sonogashira reaction 
该催化剂可以很容易地从反应体系分离利用, 循环使用 多次而无显著的活性降低.

2014 年, Wang 课题组 ${ }^{[66]}$ 制备了 1,2,3-三唑配体修饰 的磁性纳米粒子, 并用于催化吲哚及其衍生物和芳基嗍 酸类化合物的反应, 取得了令人满意的效果, 为吲哚 2 位芳基化提供了有效合成路径(Scheme 21). 值得指出 的是, 磁性纳米 $\mathrm{Fe}_{3} \mathrm{O}_{4}$ 上的催化剂可以很好地回收和再 利用，其催化活性并没有显著降低.

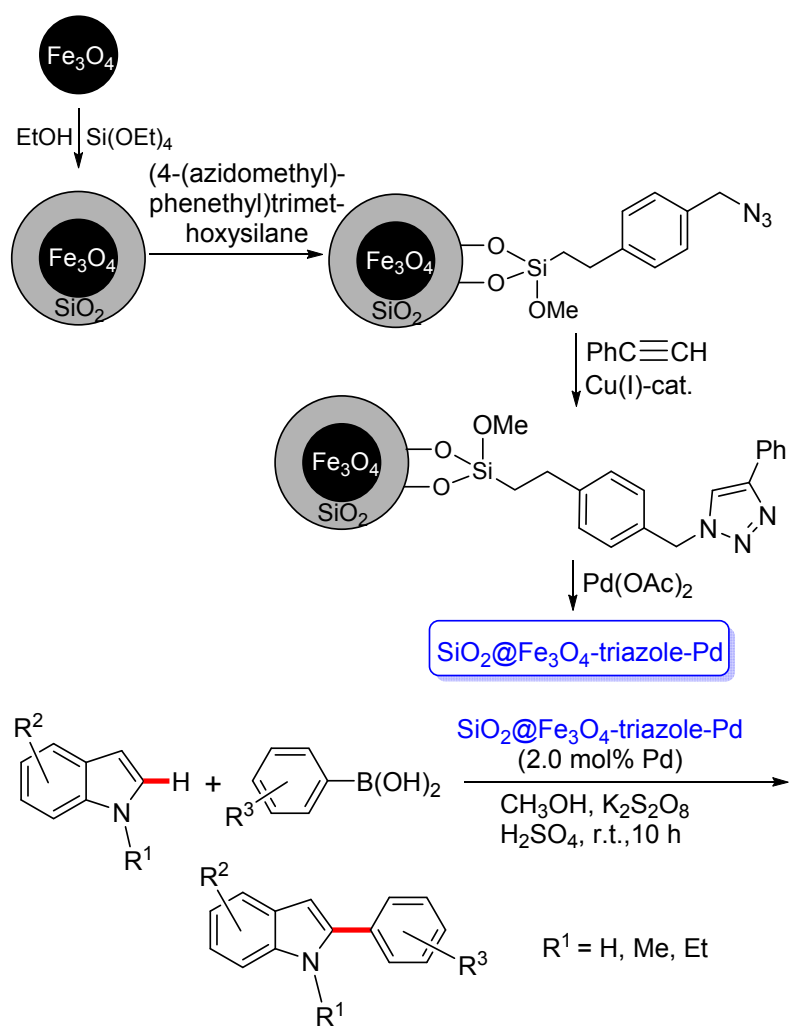

图式 21 磁性纳米粒子负载钯催化吲哚和芳基硼酸的偶联反 应

Scheme 21 Magnetic nanoparticle immobilized Pd for indoles with arylboronic acids

\section{6 总结与展望}

为解决均相催化的碳一碳键偶联反应中存在的产物 不易分离、贵金属痕量残留以及催化剂的重复使用等问 题, 通过化学家们的不解努力, 制备了不同载体的负载 型催化剂, 在一定程度上解决了上述问题, 为碳一碳键 偶联反应的工业化应用奠定了基础，同时使贵金属催化 剂的工业化应用大大降低成本, 也更有利于保护环境. 但是这些负载催化剂仍有如下不足有待进一步解决: (1) 在催化反应过程中, 有金属的流失现象; (2)载体催化剂 的活性和选择性需要提高; (3)发展负载有机小分子催化 剂 $^{[67]}$, 用于催化不对称有机反应. 因为构成生命体的绝 大多数有机分子都具有手性 ${ }^{[68]}$. 随着人们对负载催化
剂催化的碳一碳键偶联反应机理的深入研究和认识, 相 信化学家们一定设计和制备出活性与均相催化剂相婫 美且可以多次循环使用不降低活性的新型负载催化剂.

\section{References}

[1] (a) Ruan, J. W.; Xiao, J.-L. Acc. Chem. Res. 2011, 44, 614. (b) Xia, Q.-H.; Ge, H.-Q.; Ye, C.-P.; Liu, Z.-M.; Su, K.-X. Chem. Rev. 2005, 105, 1603.

(c) Ren, P.; Salihu, I.; Scopelliti, R.; Hu, X. Org. Lett. 2012, 14, 1748 .

(d) Hassan, J.; Sévignon, M.; Gozzi, C.; Schulz, E.; Lemaire, M. Chem. Rev. 2002, 102, 1359.

[2] (a) Marcial, M.-M.; Roser, P. Acc. Chem. Res. 2003, 36, 638.

(b) Yan, M.; Feng, X. Chin. J. Org. Chem. 2010, 30, 623 (in Chinese).

(颜美，冯秀娟，有机化学, 2010, 30, 623.)

(c) Leadbeater, N. E.; Marco, M. Chem. Rev. 2002, 102, 3217.

(d) Lu, J.; Toy, P. H. Chem. Rev. 2009, 109, 815.

(e) Buchmeiser, M. R. Chem. Rev. 2009, 109, 303.

(f) Bergbreiter, D. E.; Tian, J.; Hongfa, C. Chem. Rev. 2009, 109 , 530 .

[3] Köhler, K.; Wagner, M.; Djakovitch, L. Catal. Today 2001, 66, 105.

[4] Davies, I. W.; Matty, L.; Hughes, D. L.; Reider, P. J. J. Am. Chem. Soc. 2001, 123, 10139.

[5] Iyer, S.; Thakur, V. V. J. Mol. Catal. A 2000, 157, 275.

[6] Kabalka, G. W.; Pagni, R. M.; Hair, C. M. Org. Lett. 1999, 1, 1423.

[7] Melucci, M.; Barbarella, G.; Sotgiu, G. J. Org. Chem. 2002, 67, 8877.

[8] Li, X.; Yan, X.-Y.; Chang, H.-H.; Wang, L.-C.; Zhang, Y.; Chen, W.-W.; Li, Y.-W.; Wei, W.-L. Org. Biomol. Chem. 2012, 10, 495.

[9] Marck, G.; Villiger, A.; Buchecker, R. Tetrahedron Lett. 1994, 35, 3277.

[10] Köhler, K.; Heidenreich, R. G.; Krauter, J. G. E.; Pietsch, J. Chem. Eur.-J. 2002, 8, 622.

[11] Xie, X.-G; Lu, J.-P.; Chen, B.; Han, J.-J.; She, X.-G.; Pan, X.-F. Tetrahedron Lett. 2004, 45, 809.

[12] Ambulgekar, G. V.; Bhanage, B. M.; Samant, S. D. Tetrahedron Lett. 2005, 46, 2483.

[13] Chu, W.; Li, X.; Hou, Y.; Wang, H.; Li, H.; Yuan, X.; Sun, Z. Appl. Organomet. Chem. 2012, 26, 478.

[14] Liu, C.; Rao, X.; Zhang, Y.; Li, X.; Qiu, J.; Jin, Z. Eur. J. Org. Chem. 2013, 4345 .

[15] Terasawa, M.; Kaneda, K.; Imanaka, T.; Teranishi, S. J. Organomet. Chem. 1978, 162, 403.

[16] Zhang, Z. Y.; Pan, Y.; Hu, H. W.; Kao, T. Y. Synthesis 1991, 539.

[17] Schwarz, J.; Bohm, V. P. W.; Gardiner, M. G.; Grosche, M.; Herrmann, W. A.; Hieringer, W.; Raudaschl-Sieber, G. Chem. Eur.-J. 2000, 6, 1773.

[18] Lin, K. H.; Song, M. P.; Cai, D. M.; Hao, X. Q.; Wu, Y. J.; Tetrahedron Lett. 2003, 44, 3955.

[19] Yamada, Y. M. A.; Takeda, K.; Takahashi, H.; Ikegami, S.; Tetrahedron Lett. 2003, 44, 2379.

[20] (a) Li, P.; Wang, L.; Zhang, Y.; Wang, M. Tetrahedron Lett. 2008, 49, 6650 .

(b) Synfacts 2009, 2009(1), 0104.

[21] (a) Li, P.; Zhang, Y.; Wang L. Chem. Eur.-J. 2009, 15, 2045.

(b) Zhang, Y.; Li, P.; Wang, M.; Wang, L. J. Org. Chem. 2009, 74, 4364.

[22] Houdayer, A.; Schneider, R.; Billaud, D.; Ghanbaja, J.; Lambert, J. Synth. Met. 2005, 151, 165 . 
[23] Yang, Y. F.; Zhou, R. X.; Zhao, S. F.; Li, Q. L.; Zheng, X. M. J. Mol. Catal. A 2003, 192, 303.

[24] Jang, S.-B. Tetrahedron Lett. 1997, 38, 1793.

[25] Li, Y.; Hong, X. M.; Collard, D. M.; El-Sayed, M. A. Org. Lett. 2000, 2, 2385.

[26] Uozumi, Y.; Nakai, Y. Org. Lett. 2002, 4, 2997.

[27] Liu, Y.; Khemtong, C.; Hu, J. Chem. Commun. 2004, 398.

[28] Wu, L.; Li, B.-L.; Huang, Y.-Y.; Zhou, H.-F.; He, Y.-M.; Fan, Q.-H. Org. Lett. 2006, 8, 3605.

[29] Yang, J.; Li, P.; Wang, L. Synthesis 2011, 1295.

[30] Razler, T. M.; Hsiao, Y.; Qian, F.; Fu, R.; Khan, R. K.; Doubleday, W. J. Org. Chem. 2009, 74, 1381.

[31] Han, J.; Liu, Y.; Guo, R. J. Am. Chem. Soc. 2009, 131, 2060.

[32] Zhou, W.-J.; Wang, K.-H.; Wang, J.-X.; Huang, D.-F. Eur. J. Org. Chem. 2010, 416.

[33] Wang, L.; Li, P.; Zhang, Y. Chem. Commun. 2004, 514.

[34] Li, P.; Wang, L.; Li, H. Tetrahedron 2005, 61, 8633.

[35] Audic, N.; Clavier, H.; Mauduit, M.; Guillemin, J. C. J. Am. Chem. Soc. 2003, 125, 9248

[36] Corma, A.; García, H.; Leyva, A. Tetrahedron 2004, 60, 8553.

[37] Zhao, D.; Fei, Z.; Geldbach, T. J.; Scopelliti, R.; Dyson, P. J. J. Am. Chem. Soc. 2004, 126, 15876.

[38] Wang, R.; Piekarski, M. M.; Shreeve, J. M. Org. Biomol. Chem. 2006, 4, 1878

[39] Wang, R.; Xiao, J.-C.; Twamley, B.; Shreeve, J. M. Org. Biomol. Chem. 2007, 5, 671

[40] Zhou, L.; Wang, L. Synthesis 2006, 2653.

[41] Li, H.; Wang, L. Eur. J. Org. Chem. 2006, 5099.

[42] Lombardo, M.; Chiarucci, M.; Trombini, C. Green Chem. 2009, 11, 574.

[43] Wang, L.; Li, H.; Li, P. Tetrahedron 2009, 65, 364.

[44] Zhou, C.; Wang, J.; Li, L.; Wang, R.; Hong, M. Green Chem. 2011, 13,2100 .

[45] Li, P.; Wang, L. Adv. Synth. Catal. 2006, 348, 681.

[46] Zhang, L.; Wang, L.; Li, H.; Li, P. Synth. Commun. 2008, 38, 1498.

[47] Zhang, L.; Li, P.; Wang, L. Lett. Org. Chem. 2006, 3, 282.

[48] Miao, T.; Wang, L. Synthesis 2008, 363.

[49] Miao, T.; Wang, L. Tetrahedron Lett. 2007, 48, 95.

[50] Wang, Z.; Wang, L.; Li, P. Synthesis 2008, 1367.

[51] Wang, Z.; Wang, L.; Yan, J. Chin. J. Chem. 2008, 26, 1721.

[52] (a) Wang, M.; Li, P.; Wang, L. Eur. J. Org. Chem. 2008, 2255. (b) Li, P.; Wang, L. Tetrahedron 2007, 63, 5455.
[53] Li, P.; Wang, L.; Zhang, Y. Tetrahedron 2008, 64, 10825.

[54] Chen, W.; Li, P.; Wang, L. Tetrahedron 2011, 67, 318.

[55] Cai, M.; Sha, J.; Xu, Q. Tetrahedron 2007, 63, 4642.

[56] Zhao, H.; Yin, L.; Cai, M. Eur. J. Org. Chem. 2013, 1337.

[57] (a) Polshettiwar, V.; Luque, R.; Fihri, A.; Zhu, H.; Bouhrara, M.; Basset, J. M. Chem. Rev. 2011, 111, 3036.

(b) Polshettiwar, V.; Varma, R. S. Green Chem. 2010, 12, 743.

(c) Shylesh, S.; Schunemann, V.; Thiel, W. R. Angew. Chem., Int. Ed. 2010, 49, 3428.

(d) Lu, A. H.; Salabas, E. L.; Schuth, F. Angew. Chem., Int. Ed. 2007, 46, 1222.

[58] Zheng, Y.; Stevens, P. D.; Gao, Y. J. Org. Chem. 2006, 71, 537.

[59] Baruwati, B.; Guin, D.; Manorama, S. V. Org. Lett. 2007, 9, 5377.

[60] Jin, M. J.; Lee, D. H. Angew. Chem., Int. Ed. 2010, 49, 1119.

[61] Li, P.; Wang, L.; Zhang, L.; Wang, G. Adv. Synth. Catal. 2012, 354 , 1307.

[62] Zhang, L.; Li, P.; Li, H.; Wang, L. Catal. Sci. Technol. 2012, 2, 1859.

[63] Zhang, Q.; Su, H.; Luo, J.; Wei, Y. Catal. Sci. Technol. 2013, 3, 235.

[64] Zolfigol, M. A.; Khakyzadeh, V.; Moosavi-Zare, A. R.; Rostami, A.; Zare, A.; Iranpoor, N.; Beyzavie, M. H.; Luque, R. Green Chem. 2013, 15, 2132.

[65] Esmaeilpoura, M.; Sardarian, A. R.; Javidi, J. J. Organomet. Chem. 2014, 749, 233.

[66] Zhang, L.; Li, P.; Liu, C.; Yang, J.; Wang, M.; Wang, L. Catal. Sci. Technol. 2014, 4, 1979

[67] (a) Zhou, L.; Wang, L. Chem. Lett. 2007, 36, 628.

(b) Mata, Y.; Pàmies, O.; Diéguez, M. Chem. Eur.-J. 2007, 13, 3296.

(c) Miao, T.; Wang, L. Tetrahedron Lett. 2008, 49, 2173.

(d) Mata, Y.; Diéguez, M.; Pàmies, O.; Claver, C. Org. Lett. 2005, 7, 5597

[68] (a) List, B.; Lerner, R. A.; Barbas, III, C. F. J. Am. Chem. Soc. 2000, 122, 2395.

(b) Li, P.; Wang, L.; Wang, M.; Zhang, Y. Eur. J. Org. Chem. 2008, 1157.

(c) Cortez, G. S.; Tennyson, R. L.; Romo, D. J. Am. Chem. Soc. 2001, 123, 7945 .

(d) Li, P.; Wang, L.; Zhang, Y.; Wang, G.-W. Tetrahedron 2008, 64, 7633. 